\title{
IMF radial gradients in most massive early-type galaxies.
}

\author{
F. La Barbera ${ }^{1 \star}$, A., Vazdekis ${ }^{2,3}$, I. Ferreras ${ }^{4}$, A. Pasquali $^{5}$, C. Allende Prieto ${ }^{2,3}$, \\ I. Martín-Navarro ${ }^{6}$, D. S. Aguado ${ }^{7}$, R. R. de Carvalho ${ }^{8,9}$, S. Rembold ${ }^{10}$, \\ J. Falcón-Barroso ${ }^{2,3}$, G. van de Ven ${ }^{11}$ \\ ${ }^{1}$ INAF-Osservatorio Astronomico di Capodimonte, sal. Moiariello 16, Napoli, 80131, Italy \\ ${ }^{2}$ Instituto de Astrofísica de Canarias, Calle Vía Láctea s/n, E-38205 La Laguna, Tenerife, Spain \\ ${ }^{3}$ Departamento de Astrofísica, Universidad de La Laguna (ULL), E-38206 La Laguna, Tenerife, Spain \\ ${ }^{4}$ Mullard Space Science Laboratory, University College London, Holmbury St Mary, Dorking, Surrey RH5 6NT, UK \\ ${ }^{5}$ Astronomisches Rechen-Institut, Zentrum für Astronomie, Universität Heidelberg, Mönchhofstr. 12-14, D-69120 Heidelberg, Germany \\ 6 University of California Observatories, 1156 High Street, CA-95064, Santa Cruz, USA \\ 7 Institute of Astronomy, University of Cambridge, Madingley Road, CB3 OHA Cambridge, UK \\ 8 NAT - Universidade Cruzeiro do Sul / Universidade Cidade de Saõ Paulo \\ 9 Instituto Nacional de Pesquisas Espaciais (INPE) \\ 10 Departamento de F ísica, CCNE, Universidade Federal de Santa Maria, 97105-900, Santa Maria, RS, Brazil \\ 11 Department of Astrophysics, University of Vienna, Türkenschanzstrasse 17, 1180 Vienna, Austria
}

\begin{abstract}
Using new long-slit spectroscopy obtained with X-Shooter at ESO-VLT, we study, for the first time, radial gradients of optical and Near-Infrared IMF-sensitive features in a representative sample of galaxies at the very high-mass end of the galaxy population. The sample consists of seven early-type galaxies (ETGs) at $\mathrm{z} \sim 0.05$, with central velocity dispersion in the range $300 \lesssim \sigma \lesssim 350 \mathrm{~km} \mathrm{~s}^{-1}$. Using state-of-art stellar population synthesis models, we fit a number of spectral indices, from different chemical species (including TiO's and $\mathrm{Na}$ indices), to constrain the IMF slope (i.e. the fraction of low-mass stars), as a function of galactocentric distance, over a radial range out to $\sim 4 \mathrm{kpc}$. ETGs in our sample show a significant correlation of IMF slope and surface mass density. The bottom-heavy population (i.e. an excess of low-mass stars in the IMF) is confined to central galaxy regions with surface mass density above $\sim 10^{10} \mathrm{M}_{\odot} \mathrm{kpc}^{-2}$, or, alternatively, within a characteristic radius of $\sim 2 \mathrm{kpc}$. Radial distance, in physical units, and surface mass density, are the best correlators to IMF variations, with respect to other dynamical (e.g. velocity dispersion) and stellar population (e.g. metallicity) properties. Our results for the most massive galaxies suggest that there is no single parameter that fully explains variations in the stellar IMF, but IMF radial profiles at $z \sim 0$ rather result from the complex formation and mass accretion history of galaxy inner and outer regions.
\end{abstract}

Key words: galaxies: stellar content - galaxies: fundamental parameters - galaxies: formation - galaxies: elliptical and lenticular, $\mathrm{cD}$

\section{INTRODUCTION}

Our current understanding of galaxy formation and evolution rests on a framework based on a $\Lambda$ CDM expanding Universe, where the mass budget is dominated by dark matter, but where the direct observables originate from baryonic material, most notably stars. The creation of stars from gas proceeds through a complex set of physical mechanisms. One of the fundamental pieces of the

* E-mail: francesco.labarbera@inaf.it (FLB) star formation puzzle is the distribution of stellar masses at birth, i.e. the initial mass function (IMF). From basic principles, it is possible to construct arguments that can explain the overall shape of the IMF and its potential variations, via either analytic models (Hopkins 2013; Chabrier, Hennebelle, \& Charlot 2014), or more detailed hydrodynamical simulations (Padoan \& Nordlund 2002; Bate \& Bonnell 2005; Krumholz et al. 2016). However, it is through observations that we can discriminate against the different scenarios laid before us by the theoretical work. Observational constraints of the IMF have been 
sought for a long time (see Bastian, Covey, \& Meyer 2010, for an overview), including both resolved (Geha et al. 2013) and unresolved populations (see, e.g., Faber \& French 1980; Cenarro et al. 2003).

The traditionally adopted universality of the IMF has been challenged both on star-forming and quiescent systems (see Hopkins 2018, for a recent review). Regarding the latter, improved technology and population synthesis modeling have allowed us to explore a decades-long idea of using spectral line strengths that are sensitive to the giant vs dwarf stellar ratio as a discriminant of the IMF in passive populations (Spinrad \& Taylor 1971). This is especially relevant, as massive, quiescent galaxies reveal a rather extreme formation history typically described by an early, intense and short-lived formation process (see, e.g., de La Rosa et al. 2011). Such formation implies substantially different physical properties in the interstellar medium, that could, perhaps, lead to a qualitatively different mode of star formation in these systems. The finding of a bottom-heavy IMF in massive early-type galaxies (hereafter ETGs, Cenarro et al. 2003; van Dokkum \& Conroy 2010), later confirmed with independent data and analyses (see, e.g., Ferreras et al. 2013, hereafter F13; La Barbera et al. 2013, hereafter LB13; Spiniello et al. 2014) was consistent with this scenario. These results were supported by dynamical constraints, produced at around the same time, and based on kinematic studies of integral field unit data of nearby ETGs (Cappellari et al. 2012, 2013), that revealed high values of the stellar $\mathrm{M} / \mathrm{L}$ with respect to the expectations from a standard, Milky Way IMF (see also Tortora et al. 2013). Gravitational lensing over galaxy scales provides a third, independent, probe of the IMF in massive ETGs (Treu et al. 2010; Smith \& Lucey 2013).

Subsequent studies focused on radial gradients of the IMF of ETGs (Martín-Navarro et al. 2015a,b; La Barbera et al. 2016, 2017; Zieleniewski et al. 2017; van Dokkum et al. 2017; Parikh et al. 2018; Sarzi et al. 2018). These studies revealed that the non-standard, bottom heavy IMF is only found in the central regions of the most massive galaxies. At face value, massive galaxies are therefore made up of two different types of stellar populations, produced in substantially different modes of star formation. This interpretation aligns with the paradigm of a two-stage formation process (Oser et al. 2010), whereby the stellar populations of massive galaxies can be split into a component formed in-situ, mostly during the first, intense phases of formation, and an ex-situ component contributed by mergers. Regarding the in-situ phase, the massive "red nuggets" found at high redshift (see, e.g., Daddi et al. 2005; Trujillo et al. 2007; van Dokkum et al. 2008; Damjanov et al. 2011), suggest that in these systems, a gas mass $\gtrsim 10^{11} \mathrm{M}_{\odot}$ must have been transformed into stars within a $\mathrm{R} \sim 1-2 \mathrm{kpc}$ region in, roughly, a dynamical time ( 1 Gyr), therefore leading to a sustained star formation rate $\gtrsim 100 \mathrm{M}_{\odot} \mathrm{yr}^{-1}$. Such an extreme environment is bound to produce highly supersonic turbulence in the gas, resulting in fragmentation over small scales, therefore favouring a bottom-heavy IMF (Padoan \& Nordlund 2002; Hopkins 2013; Chabrier, Hennebelle, \& Charlot 2014; but see also Bertelli Motta et al. 2016).

Intriguingly, observations also find that in strongly star-forming systems, the IMF is, rather, top-heavy, i.e. producing an excess of high-mass stars with respect to the Milky Way standard (Gunawardhana et al. 2011). This conundrum can be solved by invoking a timedependent IMF whereby a strongly star forming system starts with a top-heavy IMF, followed by a later phase where a large amount of gas is locked into low-mass stars (Vazdekis et al. 1996, 1997; Davé 2008). This scenario is admittedly contrived, but it is shown to produce compatible chemical enrichment properties, X-ray binary fractions, as well as the IMF signatures found in massive ETGs (Weidner et al. 2013; Ferreras et al. 2015). The suggested claim of a potential correlation between IMF and metallicity (Martín-Navarro et al. 2015b, hereafter MN15b) gave rise to a number of modeling attempts to understand such a trend (Clauwens, Schaye, \& Franx 2016), including detailed hydrodynamical simulations (Gutcke \& Springel 2019) or the concept of the Integrated Galactic IMF (Jeřábková et al. 2018). Additional models have been explored to shed light on alternative causes of IMF variations in massive ETGs, such as cosmic rays (Fontanot et al. 2018), but the final say rests on detailed, ab initio (magneto-)hydrodynamical simulations under the physical conditions expected during the early formation of the massive cores of the ETGs we see today.

In spite of the advances made over the past few years on the issue of line strength/spectral fitting constraints on the IMF of ETGs, a number of key issues remain controversial. The interpretation of the spectral features is still open to debate, as, for instance, the spectral indices targeted to discriminate between giants and low mass dwarfs in evolved populations is prone to degeneracies, with respect to age, and most notably, chemical composition. For instance, some claims were made that the strong $\mathrm{Na}$ I-dependent feature found at $\lambda \sim 0.82 \mu \mathrm{m}$ could be partly, or even fully, described by an overabundant $[\mathrm{Na} / \mathrm{Fe}]$ (Jeong et al. 2013; McConnell et al. 2016). Such a problem is overcome by the joint analysis of a battery of spectral features with different sensitivity to specific chemical abundance ratios (see, e.g., Spiniello et al. 2012; La Barbera et al. 2013), and by a detailed analysis of a number of Na-sensitive line strengths over a wide spectral window (La Barbera et al. 2017). Moreover, the finding of massive ETGs with apparently standard IMFs, but selected as strong gravitational lenses (Smith \& Lucey 2013; Smith et al. 2015; Smith, Lucey \& Conroy 2015; Leier et al. 2016) poses additional questions as to why these galaxies behave differently to the general population of massive ETGs found in SDSS-based samples involving thousands of spectra (Newman et al. 2017).

This paper completes the study of a sample of massive ETGs observed with the X-Shooter instrument at the European Southern Observatory-Very Large Telescope (Vernet et al. 2011). This project was aimed at exploring in detail the radial gradients of the IMF in a carefully defined set of ETGs with very high velocity dispersion - where the departure from a standard IMF is expected to be highest - taking advantage of the wide spectral coverage allowed by the X-Shooter spectrograph, as well as the latest, stateof-the-art models of stellar population synthesis, coupled to theoretical models of stellar atmospheres to decipher the role of chemical composition in the derivation of the IMF. In La Barbera et al. (2016, hereafter LB16), we analyzed the 
radial IMF gradient of one of the targeted ETGs (XSG1), based on $\mathrm{TiO}$ spectral features and the Wing-Ford band, finding robust trends regarding the IMF, along with further constraints on the shape of the mass function at the very low mass end. In La Barbera et al. (2017, hereafter LB17), we extended the analysis by including four Na-sensitive indices that cover a wide spectral range (between $\lambda \sim 0.6$ and $2.2 \mu \mathrm{m}$ ), and targeted two ETGs, confirming previous results of IMF variations regardless of the $[\mathrm{Na} / \mathrm{Fe}]$ chemistry. This paper presents a comprehensive analysis of the full data set, comprising seven ETGs, with the full spectral range of XShooter allowing us to target a comprehensive set of line strengths.

The paper is structured as follows. In Sec. 2, we describe our sample of massive ETGs (hereafter XSG sample), and the new X-Shooter data. Sec. 3 describes the stellar population models used to analyze the spectra. The approach to perform the stellar population study is detailed in Sec. 4, while Sec. 5 presents the results. A discussion follows in Sec. 6.

\section{SAMPLE AND NEW DATA}

Our sample consists of seven massive ETGs. Six galaxies have been selected from the pool of most massive ETGs at the lowest redshift limit $(z \sim 0.05)$ of the SPIDER survey (La Barbera et al. 2010a, hereafter SpiderI), while one extra ETG (named XSG10, see below) has been selected from SDSS-DR7 applying the same criteria as SPIDER ETGS, but at slightly lower redshift $(z \sim 0.048)$ than the SPIDER sample $(0.05 \leqslant z \leqslant 0.095)$. In F13 and LB13, we have used the highest-quality SDSS spectra of SPIDER ETGs to construct a subsample of 18 stacked spectra ${ }^{1}$, within narrow bins of central velocity dispersion, $\sigma$, each bin with a width of $10 \mathrm{~km} \mathrm{~s}^{-1}$, except for the last two, with $260 \leqslant \sigma \leqslant 280$, and $280 \leqslant \sigma \leqslant 320 \mathrm{~km} \mathrm{~s}^{-1}$, respectively. All targets (including XSG10) analyzed in the present work have $\sigma$ in the range of the highest velocity dispersion bin (from 280 to $320 \mathrm{~km} \mathrm{~s}^{-1}$ ) defined in LB13, and have been selected based on line-strengths of IMF-sensitive absorption features and abundance ratio estimates from the SDSS spectra. In particular, in order to probe the range of values in the parent $\sigma$ bin, ETGs in our sample have SDSS estimates of $[\mathrm{Mg} / \mathrm{Fe}]$ in the range from $\sim 0.3$ to $\sim 0.5$ dex (with a typical uncertainty of 0.1 dex; see LB13), and SDSS measurments of $\mathrm{TiO} 2(\mathrm{NaD})$ in the range from $\sim 0.08$ to $\sim 0.095 \mathrm{mag}$ (4.5 to $5.5 \AA$ ). Therefore, our sample should be representative of the high-mass end population of ETGs. Throughout the present work, we refer to our targets as XSG1, XSG2, XSG6 ${ }^{2}$, XSG7, XSG8, XSG9, and XSG10, respectively. Basic galaxy properties, including the SDSS identification of

1 Notice that the 18 stacked spectra analyzed in F13 and LB13 are made public available at the following link: http://www.iac.es/proyecto/miles/pages/otherpredictionsdata.php.

2 Notice that other three targets, named XSG3, XSG4, and XSG5, were also included in our VLT X-Shooter observing proposals, but not observed. Therefore, they are not included in out sample. each object, are provided in Tab. 1. In App. A, we describe the environment where the XSGs reside, based on the SDSS-DR7 group catalogue of Wang et al. (2014). Most of the XSGs, i.e. XSG1, XSG6, XSG8, XSG9, and XSG10 are centrals of a galaxy group, while XSG2 and XSG7 are satellites ${ }^{3}$. Also, in App. C, we present a surface photometry analysis of the XSGs, based on the SDSS photometry. Remarkably, our analysis shows that the estimate of the effective radius, $\mathrm{R}_{\mathrm{e}}$, for most XSGs depends significantly on the method used to estimate such a quantity. Since we cannot assign a unique scale-length to XSGs' light profiles, when studying stellar population properties as a function of radius (see below), we consider galactocentric distances in units of $\mathrm{kpc}$, without rescaling them by (a given estimate of) the effective radius. We also discuss the effect of rescaling the IMF profiles with $R_{e}$ in App. D, and we come back on this point in Sec. 5 .

\subsection{Observations and data reduction}

For all seven targets, we have obtained new, deep longslit spectroscopy with the X-Shooter spectrograph at the ESO-VLT, on Cerro Paranal (Proposal IDs: 092.B-0378, 094.B-0747; 097.B-0229; PI: FLB). X-Shooter is a secondgeneration ESO-VLT instrument - a slit echelle spectrograph that covers a wide spectral range (3000-25000 $\mathrm{A}$ ), at relatively high resolution (Vernet et al. 2011). The spectral range is covered by splitting the incoming bin into three independent arms, ultraviolet-blue (UVB: 3000-5900 $\mathrm{A}$ ); visible (VIS: $5300-10200 \AA$ ); and near-infrared (NIR: 9800$25000 \AA$ ). Details on the data for XSG1 and XSG2 have been provided in LB16 and LB17. We give here only a short summary. The X-Shooter slit is 11" long, with a spatial scale of $0.16 \mathrm{arcsec} / \mathrm{pixel}$ in the UVB and VIS, and $0.21 \mathrm{arcsec} / \mathrm{pixel}$ in the NIR, arms. For all observations, we adopted an instrument setup with 0.9 "-, 0.9 "-, and 1.0 "- wide slits, resulting into a resolution power of $R \sim 4400, \sim 7500$, and $\sim 5500$, in the UVB, VIS, and NIR arms, respectively. We have observed XSG1 (XSG2) through five (ten) observing blocks (OBs), each including two exposures on target, interspersed by two (one) sky exposures, with the same integration time as for the science target. This setup gives a total on-target exposure time of $\sim 1.7,1.9$, and $2.1 \mathrm{hr}$, in the UVB, VIS, and NIR arms, respectively (see LB16 and LB17 for details). XSG6, XSG7, XSG8, XSG9, and XSG10 were observed with the same setup as for XSG1. In order to minimize slit losses due to problems with the X-Shooter atmospheric dispersion corrector, observations were taken at parallactic angle, resulting into data taken at two/three position angles (depending on the galaxy) for each target. Observations were carried out in service mode, with a median seeing (as measured at the telescope) of $\sim 0.8-0.9^{\prime \prime}$ (FWHM), depending on target. Due to bad weather conditions, one (two) exposure(s) for XSG6 (XSG7) were not usable for our purposes, resulting into a slightly lower exposure time for these galaxies. For each arm, the data were pre-reduced using version 2.4.0 of the data-reduction pipeline (Modigliani et al. 2010),

3 However, as discussed in the Appendix, the classification of XSG7 is uncertain, this galaxy having similar mass of, and being very close to, the brightest group galaxy. 
Table 1. Main galaxy properties. Column 1 gives the label used throughout the present work for each galaxy. Column 2 is the galaxy SDSS ID, while columns 3 and 4 are the galaxy RA and DEC coordinates. Column 5 is the galaxy total magnitude in $\mathrm{r}$ band, obtained by averaging total magnitudes of best-fitting Sersic and B+D models (see Sec. 3 for details). Columns 6 and 7 report galaxy redshifts, as derived from our X-Shooter spectroscopy, and retrieved from the SDSS database, respectively. Columns 8 and 9 report SDSS and $\mathrm{X}$-Shooter central velocity dispersions of each galaxy, respectively.

\begin{tabular}{|c|c|c|c|c|c|c|c|c|}
\hline XSG\# & $\begin{array}{l}\text { SDSS ID } \\
\qquad(2)\end{array}$ & $\begin{array}{c}\text { RA } \\
(\mathrm{deg}) \\
(3)\end{array}$ & $\begin{array}{l}\text { DEC } \\
(\mathrm{deg}) \\
(4)\end{array}$ & $\begin{array}{c}\mathrm{M}_{\mathrm{r}} \\
(\mathrm{mag}) \\
(5)\end{array}$ & $\begin{array}{c}\mathrm{z} \\
\text { SDSS } \\
(6)\end{array}$ & $\begin{array}{c}\mathrm{z} \\
\text { X-Shooter } \\
(7)\end{array}$ & $\begin{array}{c}\sigma_{0} \\
\mathrm{SDSS} \\
\left(\mathrm{km} \mathrm{s}^{-1}\right) \\
(8)\end{array}$ & $\begin{array}{c}\sigma_{0} \\
\text { X-Shooter } \\
\left(\mathrm{km} \mathrm{s}^{-1}\right) \\
(9)\end{array}$ \\
\hline 1 & $\mathrm{~J} 142940.63+002159$ & 217.41929 & 0.366398 & -22.7 & $\begin{array}{r}0.055787 \\
\pm 0.0002\end{array}$ & $\begin{array}{c}0.055757 \\
\pm 0.000006\end{array}$ & $301 \pm 9$ & $333 \pm 3$ \\
\hline 2 & J002819.3-001446.7 & 7.08043 & -0.246338 & -22.3 & $\begin{array}{c}0.059951 \\
\pm 0.0002\end{array}$ & $\begin{array}{c}0.05992 \\
\pm 0.000007\end{array}$ & $292 \pm 11$ & $302 \pm 9$ \\
\hline 6 & $\mathrm{~J} 144120.36+104749.8$ & 220.33484 & 10.79719 & -23.3 & $\begin{array}{c}0.051279 \\
\pm 0.0002\end{array}$ & $\begin{array}{c}0.051249 \\
\pm 0.000008\end{array}$ & $286 \pm 10$ & $305 \pm 13$ \\
\hline 7 & $\mathrm{~J} 151451.68+101530.4$ & 228.71533 & 10.25845 & -22.7 & $\begin{array}{r}0.054857 \\
\pm 0.0002\end{array}$ & $\begin{array}{c}0.054843 \\
\pm 0.000009\end{array}$ & $288 \pm 12$ & $319 \pm 15$ \\
\hline 8 & J015418.07-094248.4 & 28.575312 & -9.71347 & -23.8 & $\begin{array}{c}0.052451 \\
\pm 0.0002\end{array}$ & $\begin{array}{c}0.052353 \\
\pm 0.000007\end{array}$ & $293 \pm 11$ & $332 \pm 8$ \\
\hline 9 & J005551.88-095908.3 & 13.966206 & -9.98565 & -23.7 & $\begin{array}{c}0.054750 \\
\pm 0.0002\end{array}$ & $\begin{array}{c}0.05476 \\
\pm 0.000008\end{array}$ & $296 \pm 12$ & $349 \pm 6$ \\
\hline 10 & J075354.98+130916.5 & 118.479088 & 13.15459 & -22.5 & $\begin{array}{c}0.047671 \\
\pm 0.0002\end{array}$ & $\begin{array}{c}0.04763 \\
\pm 0.000006\end{array}$ & $305 \pm 10$ & $338 \pm 4$ \\
\hline
\end{tabular}

performing the subsequent reduction steps (i.e. flux calibration, sky subtraction, and telluric correction) with dedicated FORTRAN software developed by the authors. We refer the reader to LB16 and LB17 for a detailed description of each reduction step (see also Schönebeck et al. 2014). As shown in App. B, in the galaxy central regions, our new X-Shooter data are fully consistent with the existing (optical) SDSS spectroscopy.

\subsection{Radial binning}

For each galaxy we extracted $1 \mathrm{D}$ spectra at different galactocentric distances, by summing up all available exposures, and folding up data from opposite sides of the X-Shooter slit around the galaxy photometric centre (see LB16 for details). For the latter step, each row of the two-dimensional spectrum was first corrected to restframe, using the rotation velocity profile of each galaxy, as derived with the software PPXF (Cappellari \& Emsellem 2004, see below). The kinematics of the XSGs will be presented in a forthcoming paper. For the present work, we just notice that all galaxies have little rotation velocity, less than $\sim 50(\sim 100) \mathrm{km} \mathrm{s}^{-1}$ for XSG6, XSG7, XSG8, XSG9, and XSG10 (XSG1 and XSG2; see figure 2 of LB16 for the kinematic profile of XSG1). In order to minimize seeing effects, the innermost spectra of all galaxies were extracted within an aperture of width $1.3^{\prime \prime}$ (i.e. $\pm 0.675^{\prime \prime}$ ) around the photometric centre, corresponding to a factor of 1.5 times the mean seeing FWHM of our observations. The bin size was then increased adaptively outwards, in order to ensure an high median S/N (>90) per $\AA$ in the optical spectral range (from 4800 to $5600 \AA$ ). This procedure gives six radially binned spectra for XSG1 and XSG8; five binned spectra for XSG6, XSG7, XSG9, XSG10; and four radial bins for XSG2. For XSG1, the binned spectra have been shown in figure 4 of LB16, with spectra for all the other galaxies having similar quality. In particular, the median $\mathrm{S} / \mathrm{N}$ measured in the central bins is very high, with values ranging from 170 (per $\AA$ ), for XSG9, to 270 for XSG10.

For all radially binned spectra, the velocity dispersion $\sigma$ was measured as detailed in LB16, by running PPXF on different spectral regions of the UVB and VIS X-Shooter arms $(\lambda \lambda=4000-9000 \AA)$, and combining the corresponding probability distribution functions into final estimates. For each galaxy, the velocity dispersion of the central X-Shooter spectrum, $\sigma_{0}$, is reported in Tab. 1 , together with the SDSS measurements of $\sigma$. The quoted uncertainties on $\sigma_{0}$ combine, in quadrature, the formal measurement errors with the rms of $\sigma_{0}$ estimates among different spectral regions (see above). All the XSG's have $\sigma_{0}$ larger than $300 \mathrm{~km} \mathrm{~s}^{-1}$, consistent with the selection of these objects to be very massive systems. The velocity dispersion profiles will be presented in a forthcoming paper. We just point out here that all galaxies have a shallow $\sigma$ gradient with galactocentric distance, with a drop of $\sim 40-70 \mathrm{~km} \mathrm{~s}^{-1}$ (depending on the galaxy), over the radial range probed by $\mathrm{X}$-Shooter. Because of that, the SDSS values of $\sigma_{0}$ are smaller (by $\sim 10 \%$, on average) than those estimated with X-Shooter (see the Table), as they refer to the SDSS fiber aperture of radius $1.5^{\prime \prime}$, a factor more than two larger than the size of the X-Shooter innermost radial bins (see above).

\section{STELLAR POPULATION MODELS}

\subsection{Na-EMILES SSPs}

Our analysis relies on the Na-EMILES stellar population models, a dedicated version of the EMILES models covering a range of $[\mathrm{Na} / \mathrm{Fe}]$ abundance ratios (see LB17). EMILES models cover the spectral range from 0.35 to $5 \mu \mathrm{m}$, at "moderately" high resolution (see below). Such wide wavelength range is achieved by joining different simple stellar population (SSP) model predictions based on empirical stellar libraries, namely MILES 
in the optical range (Sánchez-Blázquez et al. 2006), from $\lambda \sim 3540 \AA$ to $\lambda \sim 7410 \AA$, Indo-US (Valdes et al. 2004) and CaT (Cenarro et al. 2001a) out to $\lambda \sim$ $8950 \AA$ (Vazdekis et al. 2012), and the IRTF stellar library (Cushing et al. 2005; Rayner et al. 2009), extending the $\lambda$ range out to $5 \mu \mathrm{m}$ (see Röck et al. 2016 for details about the joining procedure). The spectral resolution is kept constant with wavelength (at FWHM $=2.5 \AA$ ) for all libraries, except for IRTF, having a constant $\sigma=60 \mathrm{~km} \mathrm{~s}^{-1}$ (see figure 8 of Vazdekis et al. 2016). The models are computed for two sets of scaled-solar theoretical isochrones, namely the ones of ? (BaSTI) and Girardi et al. (2000) (Padova00). The BaSTI isochrones are supplemented with the stellar models of Cassisi et al. (2000), which allow the very lowmass (VLM) regime to be covered down to $0.1 M_{\odot}$. The temperatures of these low-mass stars are cooler than those of Padova00 (Vazdekis et al. 2012). Both sets of isochrones include the thermally pulsing AGB regime using simple synthetic prescriptions, providing a significantly smaller contribution for this evolutionary phase at intermediateaged stellar populations in comparison to the models of Marigo et al. (2008) and Maraston (2005), and more similar to that of Bruzual \& Charlot (2003) models. Finally, there are differences between the BaSTI and Padova00 isochrones, described in detail in Cassisi et al. (2004), ?, and Vazdekis et al. (2015, hereafter V15). To cover a range in $[\mathrm{Na} / \mathrm{Fe}]$, we apply theoretical differential corrections for $[\mathrm{Na} / \mathrm{Fe}]$ overabundance, specifically computed for each individual stellar spectrum in the empirical libraries. The $\mathrm{Na}^{-}$ EMILES SSPs are then constructed, as detailed in LB17, based on scaled-solar isochrones. Notice that this approach differs from that used in V15, where we have computed $\alpha-$ (rather than $\mathrm{Na}^{-}$) enhanced models based on corrections applied directly to the model SSPs, rather than to individual stars. Also, the $\alpha$-enhanced models only cover the optical (MILES) spectral range, while Na-enhanced models are computed over the optical plus NIR spectral range (see above). We point out that the Na-EMILES models used in the present analysis differ from those presented in our previous work, in that (i) we have constructed both BaSTI and Padova00 SSPs (rather than only Padova00 models, as in LB17), (ii) the models are computed for a wider range of IMF slopes (see below); and (iii) stellar spectra have been corrected to $[\mathrm{Mg} / \mathrm{Fe}]=0$, based on $[\mathrm{Mg} / \mathrm{Fe}]$ abundances from Milone et al. (2011), using theoretical differential corrections computed for each individual stellar spectrum in the empirical libraries ${ }^{4}$.

Na-EMILES models are computed for different IMF shapes, as described in Vazdekis et al. (2003) and V15, and in particular for two power-law distributions, as defined in Vazdekis et al. (1996), i.e unimodal (single power-law) and bimodal. The lower and upper mass-cutoffs are set to 0.1 and $100 \mathrm{M}_{\odot}$, respectively. The unimodal and bimodal IMFs

${ }^{4}$ However, since the abundance pattern of stars in the empirical libraries follows that of the Galaxy (i.e. stars are alpha-enhanced at sub-solar metallicity, while they have $[\mathrm{Mg} / \mathrm{Fe}] \sim 0$ in the solar and super-solar metallicity regime), the corrections have negligible impacts on models relevant for the present work, where we study massive ETGs, whose spectra typically have super-solar metallicities. are defined by their logarithmic slope, $\Gamma$ and $\Gamma_{b}$, respectively. For reference, the Salpeter (1955) IMF is obtained when adopting a unimodal IMF with $\Gamma=1.35$, whereas the Kroupa (2001) Universal IMF is closely approximated by a bimodal IMF with $\Gamma_{b}=1.3$. The difference between unimodal and bimodal IMFs is that the bimodal distribution is smoothly tapered towards low masses $\left(\sim 0.5 M_{\odot}\right)$; hence, varying the high-mass end slope $\Gamma_{b}$ changes the dwarf-togiant ratio in the IMF through its overall normalization. While this approach is different with respect to a change of the low-mass slope (e.g. Conroy \& van Dokkum 2012b), the bimodal parameterisation is suitable for our purposes, as most IMF-sensitive features depend on the dwarf-to-giant ratio in the IMF (e.g. LB13, LB16). Since a low-mass tapered IMF provides mass-to-light ratios consistent with dynamical constraints (Lyubenova et al. 2016) and is able to describe both optical and NIR IMF-sensitive features (see LB16, where the fits with a single power-law distribution were largely disfavoured), in the present work we consider only models with a low-mass tapered distribution.

\subsection{Spectral indices}

The wide spectral range provided by X-Shooter allows us to analyze both optical and NIR spectral features simultaneously. Following the same approach as in our previous works (e.g. LB13, LB16, LB17), we constrain stellar population properties by comparing observed and model linestrengths for a selected set of optical and NIR spectral indices. For the present work, we consider the age-sensitive Balmer lines, $\mathrm{H} \beta_{\mathrm{o}}$ and $\mathrm{H} \gamma_{\mathrm{F}}$, the total metallicity indicator $[\mathrm{MgFe}]^{\prime}$, the IMF-sensitive features $\mathrm{TiO}$, $\mathrm{TiO} 2_{\mathrm{SDss}}$, aTiO, and $\mathrm{Mg} 4780$, as well as the four Na indices, NaD, NaI8190, NaI1.14, and NaI2.21 ${ }^{5}$, which are sensitive to both IMF and $\mathrm{Na}$ abundance ratio. The index definition for $\mathrm{TiO} 1, \mathrm{H} \gamma_{\mathrm{F}}$, and $\mathrm{NaD}$ is the same as in Trager et al. (1998), while $\mathrm{H} \beta_{\mathrm{o}}$ is the optimized $\mathrm{H} \beta$ index defined by Cervantes \& Vazdekis (2009). The TiO2 $2_{\mathrm{SDSS}}$ is defined as in LB13, being a modified version of $\mathrm{TiO} 2$ from Trager et al. (1998). The total-metallicity indicator $[\mathrm{MgFe}]^{\prime}$ is a combined $\mathrm{Mgb}$ and Fe index, defined by Thomas et al. (2003a) to be insensitive to $[\mathrm{Mg} / \mathrm{Fe}]$ abundance ratios (see also V15). Finally the Mg4780 is from Serven, Worthey, Briley (2005), aTiO from Spiniello et al. (2014), while NaI8190, NaI1.14, and NaI2.21 are defined as in Conroy \& van Dokkum (2012a, hereafter CvD12a), with some modifications (in the air system) as described in LB17. In a forthcoming paper, we will also explore features from other chemical species (e.g. Ca, $\mathrm{K}, \mathrm{CO}$ ) available over the wide X-Shooter spectral range, thanks to the ongoing development of dedicated stellar population models (as the Na-EMILES). Fig. 1 shows, as an example, the radially binned spectra for one galaxy in our sample, with the inset panels zooming into the spectral regions around the selected IMF-sensitive spectral features.

For each IMF slope (see Sec. 3.1), we compute predictions for single SSP models (hereafter 1SSP) as well as mod-

5 Notice that, for each spectrum, only indices not affected by sky residuals, and/or large uncertainties $(>100 \%)$ were included in the analysis. For this reason, NaI2.21 is only included in the analysis for the innermost radial bins of each galaxy (see App. E). 

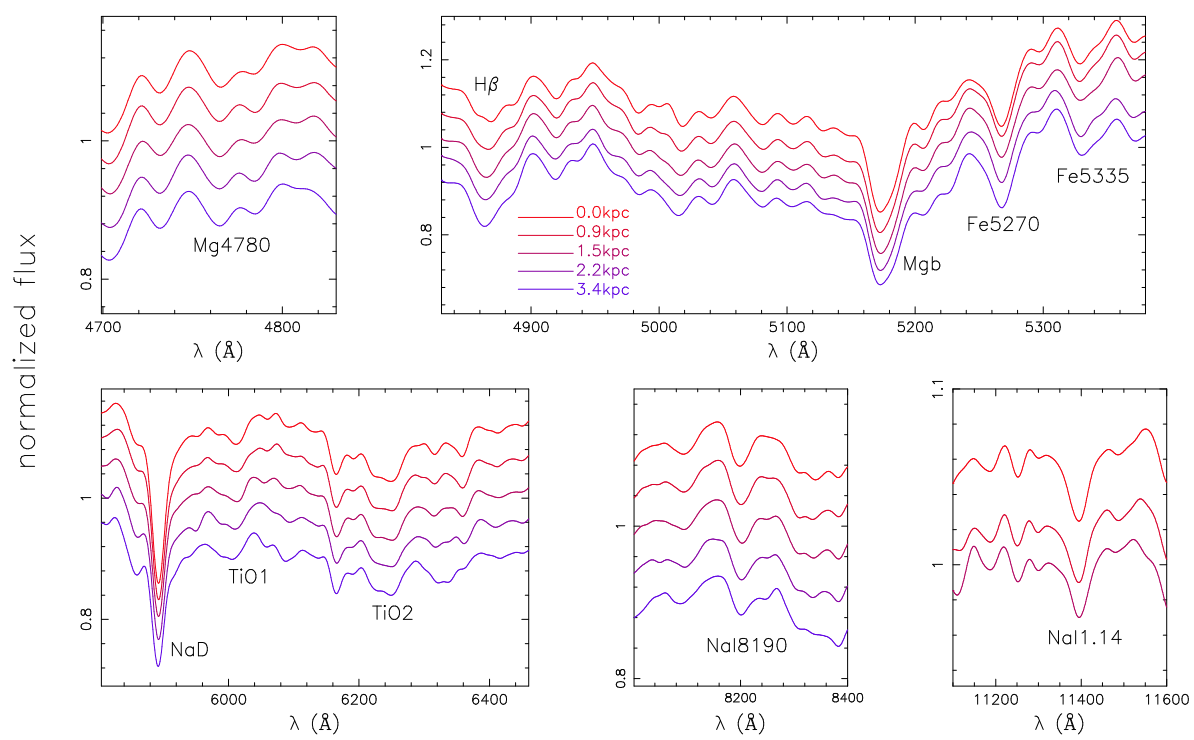
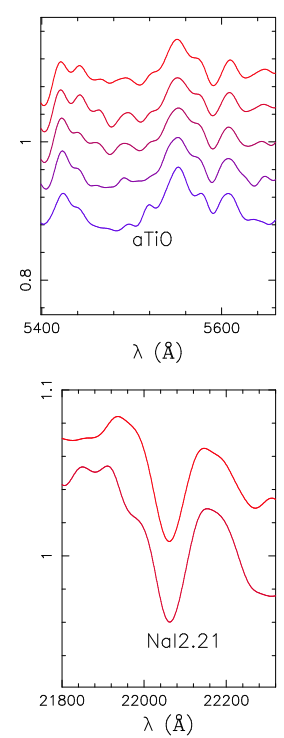

Figure 1. X-SHOOTER spectra of XSG6 (one of the new galaxies analyzed in the present work). Different panels show different spectral regions, with the main absorption features included in our analysis (see labels in each plot, and Sec. 4.1). The data are radially binned in five galacto-centric distances, out to $\mathrm{R} \sim 3.5 \mathrm{kpc}$, from the galaxy center. Different colours, from red through blue, correspond to different radial positions, as labelled in the top-middle panel. All spectra have been smoothed, for displaying purposes, to a common velocity dispersion of $400 \mathrm{~km} \mathrm{~s}^{-1}$. Notice that NaI1.14 and NaI2.21 are only included in the analysis for the innermost radial bins of each galaxy, as in the outermost spectra these indices have large uncertainties and/or are affected by sky subtraction residuals (see Sec. 3.2).

els with a fraction of young stars added on top of an old SSP (hereafter 2SSP). In the 1SSP case, we compute indices for SSPs with ages between about 4 and 14 Gyr, and metallicities between about -0.4 and $+0.2 \mathrm{dex}$, as younger/lowermetallicity SSPs are not relevant for our sample of massive ETGs. More specifically, in the case of Padova00 models, we use SSPs with ages of $\{3.5481,5.0119,6.3096,7.9433,8.9125$, $10.0000,11.2202,12.5893,14.1254\}$ Gyr, and metallicites $\{-$ $0.4,0,+0.22\}$ dex. For BaSTI models, the adopted SSPs have ages in the range from 4 to $14 \mathrm{Gyr}$, at a step of $1 \mathrm{Gyr}$, and metallicites $\{-0.35,-0.25,+0.06,+0.15,+0.26\}$ dex. Notice that BaSTI models are computed for more metallicities than the Padova00 set (see V15). In particular, BaSTI SSPs are also computed for $[\mathrm{Z} / \mathrm{H}]=0.4$ dex, but these models are unsafe according to the quality parameter defined in V15, and therefore are not used in the present work. The computed line-strengths are linearly extrapolated ${ }^{6}$ out to $[\mathrm{Z} / \mathrm{H}]=0.7$ for Padova00, and out to $0.75 \mathrm{dex}^{7}$ for BaSTI models, and then interpolated over finer grids with 111 (42) steps in age (metallicity). All index grids are computed for different values of $[\mathrm{Na} / \mathrm{Fe}]=\{0,0.3,0.6,0.9\}$, for which $\mathrm{Na}-\mathrm{EMILES}$ models have been computed. Models

6 The extrapolation is carried out by performing a linear fit of each index versus the total metallicity indicator $[\mathrm{MgFe}]^{\prime}$. The fit is done by considering only model predictions with $[\mathrm{Z} / \mathrm{H}] \geqslant 0$, for both Padova00 and BaSTI models.

7 In practice, if we consider Padova00 models, only a few spectra (i.e. the three innermost bins for XSG8, and the outermost bin for XSG6) require a significant amount of extrapolation, while for all the other cases, metallicity is within the range of the models $([\mathrm{Z} / \mathrm{H}] \leqslant+0.22)$, or requires a small amount of extrapolation (by less than $\sim 0.1$ dex). Therefore, none of our conclusions is affected by the extrapolation procedure. with $[\mathrm{Na} / \mathrm{Fe}]=1.2$ are also computed, as in LB16, but are not used for the present analysis, as we verified a posteriori that none of our galaxy requires such extreme $[\mathrm{Na} / \mathrm{Fe}]$ abundance ratios (see Sec. 5.2). Index grids are finally interpolated with a finer step of $0.01 \mathrm{dex}$ in $[\mathrm{Na} / \mathrm{Fe}]$.

In the 2SSP case, we compute indices for linear combinations of two SSPs, with the "old" SSP having the same values of age, metallicity, IMF slope, and $[\mathrm{Na} / \mathrm{Fe}]$ as in the 1SSP case, and a younger SSP, whose age is varied between 1 and 4 Gyr, for three metallicities of $\{-0.4,0,+0.2\}(\{-0.35$, $+0.06,+0.26\}$ ) for Padova00 (BaSTI) models. The light fraction of the young to old component is varied between 1 to $15 \%^{8}$.

Notice that in order to maximize the information provided by the data, we do not smooth all spectra and models at the same sigma, but instead, for each spectrum (i.e. each galaxy/radial bin), we compare observed line-strengths to model predictions computed at the same sigma as the given spectrum (see Sec. 5.2).

\section{ANALYSIS}

\subsection{Fitting procedure}

To constrain the IMF, we adopt a similar approach to that in LB16, updated to fit $\mathrm{Na}$ absorption lines as in LB17. Overall, the method follows the approach described in our previous works (e.g. F13, LB13, La Barbera, Ferreras, \& Vazdekis 2015; Martín-Navarro et al. 2015a, MN15b, Martín-Navarro et al.

8 We verified, a posteriori, that none of our fits requires a fraction of young-to-old stars larger than $15 \%$. 
2015c). For each spectrum, we minimize the following expression,

$$
\begin{gathered}
\chi^{2}\left(\text { Age },[\mathrm{Z} / \mathrm{H}], \Gamma_{\mathrm{b}},[\mathrm{Na} / \mathrm{Fe}]\right)=\sum_{\mathrm{i}}\left[\frac{\mathrm{E}_{\mathrm{obs}, \mathrm{i}}^{\mathrm{ss}}-\mathrm{E}_{\mathrm{mod}, \mathrm{i}}}{\sigma_{\mathrm{E}_{\mathrm{obs}, \mathrm{i}}^{\mathrm{ss}}}}\right]^{2}+ \\
\sum_{\mathrm{j}}\left(\mathrm{Na}_{\mathrm{obs}, \mathrm{j}}-\mathrm{Na}_{\mathrm{mod}, \mathrm{j}}-\alpha_{\mathrm{Na}_{\mathrm{j}}} \cdot \mathrm{Na}_{\mathrm{mod}, \mathrm{j}} \cdot[\alpha / \mathrm{Fe}]\right)^{2} \cdot \sigma_{\mathrm{j}}{ }^{-2}
\end{gathered}
$$

where the index $j$ runs over $\mathrm{Na}$ features, while the index $i$ runs over the remaining selected spectral features (see Sec. 3.2 and Sec. 4.2); $\mathrm{E}_{\text {mod,i }}$ and $\mathrm{Na}_{\text {mod,j }}$ are line-strength predictions for a given (either 1SSP or 2SSP) model, $\mathrm{E}_{\mathrm{obs}, \mathrm{i}}^{\mathrm{ss}}$ are observed line-strengths $-\mathrm{E}_{\mathrm{obs}, \mathrm{i}}-$ corrected to solar-scale as detailed in LB16, while $\mathrm{Na}_{\mathrm{obs}, \mathrm{j}}$ are observed line-strengths for $\mathrm{Na}$ features. The $[\alpha / \mathrm{Fe}]$ is the $\alpha$-element abundance ratio for the given spectrum, estimated through the solar-scale proxy $\left[\mathrm{Z}_{\mathrm{Mg}} / \mathrm{Z}_{\mathrm{Fe}}\right]$ (see LB13 for details), while $\alpha_{\mathrm{Na}}$ is the normalized response $\left(\delta \mathrm{Na}_{\mathrm{J}} / \mathrm{Na}_{\mathrm{j}}\right)$ of the $\mathrm{j}$-th $\mathrm{Na}$-sensitive index to $[\alpha / \mathrm{Fe}]$. The $\alpha_{\mathrm{Na}_{\mathrm{j}}}$ have been constrained, empirically, in LB17 (see figure 5 in that paper), being consistent, in the optical, with theoretical predictions from $\alpha$-MILES models. The terms $\sigma_{\mathrm{E}_{\mathrm{obs}, \mathrm{i}}^{\mathrm{ss}}}$ and $\sigma_{\mathrm{j}}$ are the uncertainties on solar-scale corrected line-strengths and $\mathrm{Na}$ line-strengths, respectively. The minimization of Eq. 1 is performed over a discrete index grid of $\mathrm{Na}$-EMILES model predictions, with varying Age and $[\mathrm{Z} / \mathrm{H}]$ (for $1 \mathrm{SSP}$ models), $\Gamma_{\mathrm{b}}$ and $[\mathrm{Na} / \mathrm{Fe}]$ abundance ratios. For 2SSP models, the grid includes three extra parameters, given by the Age and $[\mathrm{Z} / \mathrm{H}]$ of the young component, as well as the relative light fraction of the young vs. old components (see Sec. 3). In the model grid, the effect of varying $[\mathrm{Na} / \mathrm{Fe}]$ is considered only for Na features, as for the other features, the (overall) effect of abundance ratios is already taken into account by the empirical corrections. Uncertainties on best-fitting parameters, \{Age, $[\mathrm{Z} / \mathrm{H}]$, $\left.\Gamma_{\mathrm{b}},[\mathrm{Na} / \mathrm{Fe}]\right\}$, are obtained from $N=1000$ bootstrap iterations, where the fitting is repeated after shifting observed line strengths (as well as the $\alpha_{\mathrm{Na}_{\mathrm{j}}}$ 's) according to their uncertainties.

Notice that, besides the effect of age, metallicity, and IMF slope, $\mathrm{Na}$ features are mostly sensitive only to $[\mathrm{Na} / \mathrm{Fe}]$ and $[\alpha / \mathrm{Fe}]$ abundance ratios (see CvD12a, LB17; but see also Röck et al. 2017 for a possible contribution of [C/Fe] to NaI2.21), whose effects are taken into account by $\mathrm{Na}-$ EMILES models and the $\alpha_{\mathrm{Na}_{\mathrm{j}}}$ coefficients (from LB17), respectively. Moreover, as shown in LB17, the effects of $[\mathrm{Na} / \mathrm{Fe}]$ and IMF on $\mathrm{Na}$ features are actually coupled, with a bottom-heavy IMF boosting up the $[\mathrm{Na} / \mathrm{Fe}]$ response of the (NIR) Naj's. This coupling is also taken into account by our models, where Na-enhanced SSPs are computed for all different IMF's. For these reasons, in Eq. 1, Na features are treated in a different manner with respect to the other features, where the (cumulative) effect of abundance ratios (from different chemical species) is taken into account by our empirical correction procedure. Notice, however, that as discussed in LB16, the combined effects of abundance ratios is very small for most of the features (e.g. the TiO's) as the effect of different elements (e.g. $[\alpha / \mathrm{Fe}]$ and $[\mathrm{C} / \mathrm{Fe}]$ ) compensate each other. Therefore, our results are not driven by the empirical correction approach.

\subsection{Fitting schemes}

In order to check the robustness of our results, we consider different fitting cases, by (i) changing the set of fitted indices, (ii) exploring both 1SSP and 2SSP models, (iii) using models based on different isochrones, i.e. both BaSTI and Padova00 Na-EMILES. The different cases are summarized in Tab. 2. Case A fits 1SSP Padova00 models, including all indices (but the Balmer lines ${ }^{9}$ ), while cases B and $\mathrm{C}^{10}$ are the same as A but removing different sets of indices, i.e. the TiO's and all indices but TiO1 and $\mathrm{TiO} 2$, respectively. Case $\mathrm{D}$ is the same as A but for two SSPs, including also $\mathrm{H} \beta$ and $\mathrm{H} \gamma$ Balmer lines. Notice that in case A we do not include $\mathrm{H} \beta$ in the fit, as this index is also sensitive to IMF (see LB13), and we want to minimize any correlated variation of age and IMF slope. The effect of including the Balmer lines is explored with method D (providing very consistent results with method A, as shown in the following). Finally, case E is the same as A but changing the isochrones (i.e. BaSTI vs. Padova00). The rationale behind methods $\mathrm{B}$ and $\mathrm{C}$ is that $\mathrm{TiO} 1$ and $\mathrm{TiO} 2$ (i) are wide spectroscopic features, potentially affected by flux calibration issues (see LB16); (ii) have a prominent sensitivity to abundance ratios, such as [Ti/Fe], $[\mathrm{O} / \mathrm{Fe}]$, and $[\mathrm{C} / \mathrm{Fe}]$, affecting to less extent the other indices; and (iii) are more sensitive to temperature, than gravity (see Spiniello et al. 2014). Therefore, it is worth testing the impact of including/removing these indices from the analysis. In particular, we notice that, since $\mathrm{TiO} 1$ and $\mathrm{TiO} 2$ are sensitive to $[\mathrm{Ti} / \mathrm{Fe}$ ] (with $\mathrm{TiO} 2$ being less sensitive to $[\mathrm{Ti} / \mathrm{Fe}]$ than IMF, compared to TiO1), while aTiO and $\mathrm{Mg} 4780$ are not (the former being mostly sensitive to $[\mathrm{Fe} / \mathrm{H}]$, the latter to $[\mathrm{Mg} / \mathrm{Fe}]$ and $[\mathrm{Na} / \mathrm{Fe}]$; see LB16), the fitting scheme B is insensitive to non-solar [Ti/Fe] abundance ratios. To be consistent with our previous works (e.g. LB13 and LB16), we refer to case A as our "reference" fitting method, although none of the results are found to depend significantly on the fitting scheme.

\section{RESULTS}

\subsection{IMF radial gradients}

Fig. 2 plots the main result of the present work, i.e. the trend of IMF slope, $\Gamma_{\mathrm{b}}$, as a function of galactocentric distance, $\mathrm{R}$, in our sample of massive ETGs. Results are plotted for our reference fitting method (case A; see Tab. 2). The values of $\Gamma_{\mathrm{b}} \sim 1.3$ correspond to a Kroupa-like IMF, and are marked with an horizontal dashed line in the Figure. All the XSGs show a negative IMF radial gradient, with a bottom-heavy distribution in the galaxy central regions $(R \lesssim 2 \mathrm{kpc}$; see vertical dashed line in the Figure), and an IMF closer to a

9 Balmer lines (in particular $\mathrm{H} \beta$ ) are also sensitive to IMF, individual abundance ratios (e.g. $[\mathrm{C} / \mathrm{Fe}]$ ), and small fractions of young stars (see LB13, LB16, and references therein). Hence, any age determination based on $\mathrm{H} \beta$ might bias the (simultaneous) inference of IMF slope. For this reason, we have not included $\mathrm{H} \beta$ in method A (although we verified that including it does not affect significantly our results), but we have fully exploited the effect of fitting Balmer lines through method D, as detailed in the text.

10 Notice that in case C, Eq. 1 does not include the term with Na line-strengths. 
Table 2. Summary of different methods used to infer the IMF slope from observed line-strengths. Column 1 provides the label used to refer to each method. Column 2 reports the number of SSPs considered in the fitting procedure, while column 3 reports the models' isochrones, with labels iP and iT referring to Padova00 and BaSTI isochrones, respectively (see Sec.3). Column 4 gives the list of indices used for each method.

\begin{tabular}{|c|c|c|c|}
\hline $\begin{array}{l}\text { Method } \\
\text { (1) }\end{array}$ & $\begin{array}{l}\text { number of SSPs } \\
(2)\end{array}$ & $\begin{array}{c}\text { isochrones } \\
\text { (3) }\end{array}$ & $\begin{array}{l}\text { Spectral indices } \\
\text { (4) }\end{array}$ \\
\hline A & 1 & iP & {$[\mathrm{MgFe}]^{\prime}, \mathrm{TiO} 1, \mathrm{TiO} 2_{\mathrm{SDSS}}, \mathrm{aTiO}, \mathrm{Mg} 4780, \mathrm{NaD}, \mathrm{NaI} 190, \mathrm{NaI} 1.14, \mathrm{NaI} 2.21$} \\
\hline B & 1 & iP & same as method $\mathrm{A}$ but $\mathrm{w} / \mathrm{o} \mathrm{TiO} 1$ and $\mathrm{TiO} 2_{\mathrm{SDSS}}$ \\
\hline $\mathrm{C}$ & 1 & iP & {$[\mathrm{MgFe}]^{\prime}, \mathrm{TiO} 1, \mathrm{TiO}_{\mathrm{SDSS}}$ (i.e. same as $\mathrm{A}$ but $\mathrm{w} / \mathrm{o}$ aTiO, $\mathrm{Mg} 4780$, and $\mathrm{Na}$ indices) } \\
\hline $\mathrm{D}$ & 2 & iP & same as method A plus $\mathrm{H} \beta_{\mathrm{O}}$ and $\mathrm{H} \gamma_{\mathrm{F}}$ \\
\hline $\mathrm{E}$ & 1 & $\mathrm{iT}$ & same as method $\mathrm{A}$ \\
\hline
\end{tabular}

Kroupa-like distribution at larger distances. For what concerns the quality of the fits to spectral indices, we show in App. E that our models are able to match reasonably well all the observed line-strengths (for all galaxies, and radial bins). Fig. 3 also shows that our results are independent of the fitting method. In particular the $\Gamma_{\mathrm{b}}$ profiles remain virtually indistinguishable from the reference case when considering BaSTI (rather than Padova00) stellar population models, or excluding $\mathrm{Na}$ features from the fits (see upperleft and lower-right panels in the Figure, corresponding to methods $\mathrm{E}$ and $\mathrm{C}$, respectively). Negative IMF gradients are also found when excluding $\mathrm{TiO}$ features (method B; see lower-left panel) or when assuming 2SSP models (method D; see upper-right panel), although in these cases, the scatter is larger than in our reference fitting method. For method B, the scatter increases because excluding $\mathrm{TiO}$ features makes the fits more sensitive to the correlated variation of IMF slope and $[\mathrm{Na} / \mathrm{Fe}]$ abundance ratio, while in method $\mathrm{D}$, results are more sensitive to the degeneracy between age and IMF slope (due to the sensitivity of Balmer and TiO lines to both parameters; see LB13).

Figs. 2 and 3 show that the IMF radial profiles of all XSGs are very similar to each other, when plotted versus physical scale (i.e. galactocentric distance in units of $\mathrm{kpc}$ ). XSG10 is the only object with a flatter trend at $\mathrm{R} \gtrsim 1 \mathrm{kpc}$. The Pearson's correlation coefficient, $\rho$, of $\Gamma_{\mathrm{b}}$ versus $\mathrm{R}$ for the whole data-set (i.e. all galaxies and radial bins) indicates a very significant correlation, with a value of $\rho \sim-0.9$ (see Fig. 2). On the contrary, as shown in App. D, when plotting IMF profiles versus normalized galactocentric distances, $R / R_{e}$, the profiles for different galaxies do not coincide, independent of the method used to measure the effective radius (e.g. Sérsic vs. B+D fitting; see Fig. D1). Hence, a physical distance of $\sim 2 \mathrm{kpc}$ marks the characteristic scale of IMF radial variations in our sample of very massive ETGs. Since other galaxy parameters (e.g. metallicity, and abundance ratios) do also change with galactocentric distance in ETGs, the fact that IMF slope correlates with galactocentric distance, does not imply, necessarily, that distance is the main driver of IMF variations. We investigate this point further in the following sections.

Our results (Fig. 2) are qualitatively consistent with other works reporting negative IMF radial gradients in (some) ETGs (e.g. Martín-Navarro et al. 2015a; van Dokkum et al. 2017; Sarzi et al. 2018; Parikh et al.
2018; Vaughan et al. 2018) ${ }^{11}$, as well as with our previous works focusing on radial trends for XSG1 only (LB16 and LB17). However, the present work explores, for the first time, IMF radial variations at the very high mass end of the galaxy distribution, for a representative, homogeneous sample of massive ETGs. Moreover, it is important to point out that other works have mostly focused on optical (or NIR) features only, while we combine features over a wide (optical+NIR) wavelength baseline.

\subsection{Local drivers of IMF variations}

Fig. 4 plots IMF slope, $\Gamma_{\mathrm{b}}$, for our reference fitting method versus different quantities (in different panels), i.e. (from top to bottom, and left to right) velocity dispersion $(\sigma),[\mathrm{Na} / \mathrm{Fe}]$ and $[\mathrm{Mg} / \mathrm{Fe}]$ abundance ratios, total metallicity $[\mathrm{Z} / \mathrm{H}]$, and age.

\subsubsection{Radial trends of age, metallicity, and abundance ratios}

Radial trends of age, metallicity, and abundance ratios for our sample of ETGs (as plotted in Fig. 4), are generally consistent with previous studies of stellar population gradients in massive galaxies. The XSGs have rather flat age gradients, in agreement with, e.g., Martín-Navarro et al. (2018, see references therein). The only exception is XSG6, whose age values tend to become younger outwards, consistent with the fact that this galaxy has the strongest ionization pattern among all XSGs, for all radial apertures (see App. F). The $[\mathrm{Mg} / \mathrm{Fe}]$ radial gradients are generally flat, with XSG1, XSG6, XSG8, XSG9 having lower $[\mathrm{Mg} / \mathrm{Fe}]$ in the outermost bin, XSG2 and XSG7 having radially constant $[\mathrm{Mg} / \mathrm{Fe}]$ profiles for all bins, and XSG10 showing a slightly positive $[\mathrm{Mg} / \mathrm{Fe}]$ gradient. This is consistent with results of, e.g., Sánchez-Blázquez et al. 2007; Greene et al. 2015; Martín-Navarro et al. 2018; Parikh et al. 2018. In contrast, van Dokkum et al. (2017) have reported positive $[\mathrm{Mg} / \mathrm{Fe}]$

11 In contrast to these works, Alton, Smith, Lucey $(2017,2018)$ measured the J-band luminosity-weighted light from dwarf stars in the IMF, $\mathrm{f}_{\mathrm{dwarf}}$, finding very little (at the few-percent level) radial variations of $f_{d \text { warf }}$ in ETGs. We notice that this result is not inconsistent with our findings. In fact, because of our adopted (bimodal) IMF parametrization, the trends in Fig. 2 imply radial gradients in $\mathrm{f}_{\mathrm{dwarf}}$ at the level of a few percent (see the conversion between $\Gamma_{b}$ and $f_{d w a r f}$ in table C2 of Alton, Smith, Lucey 2017). 


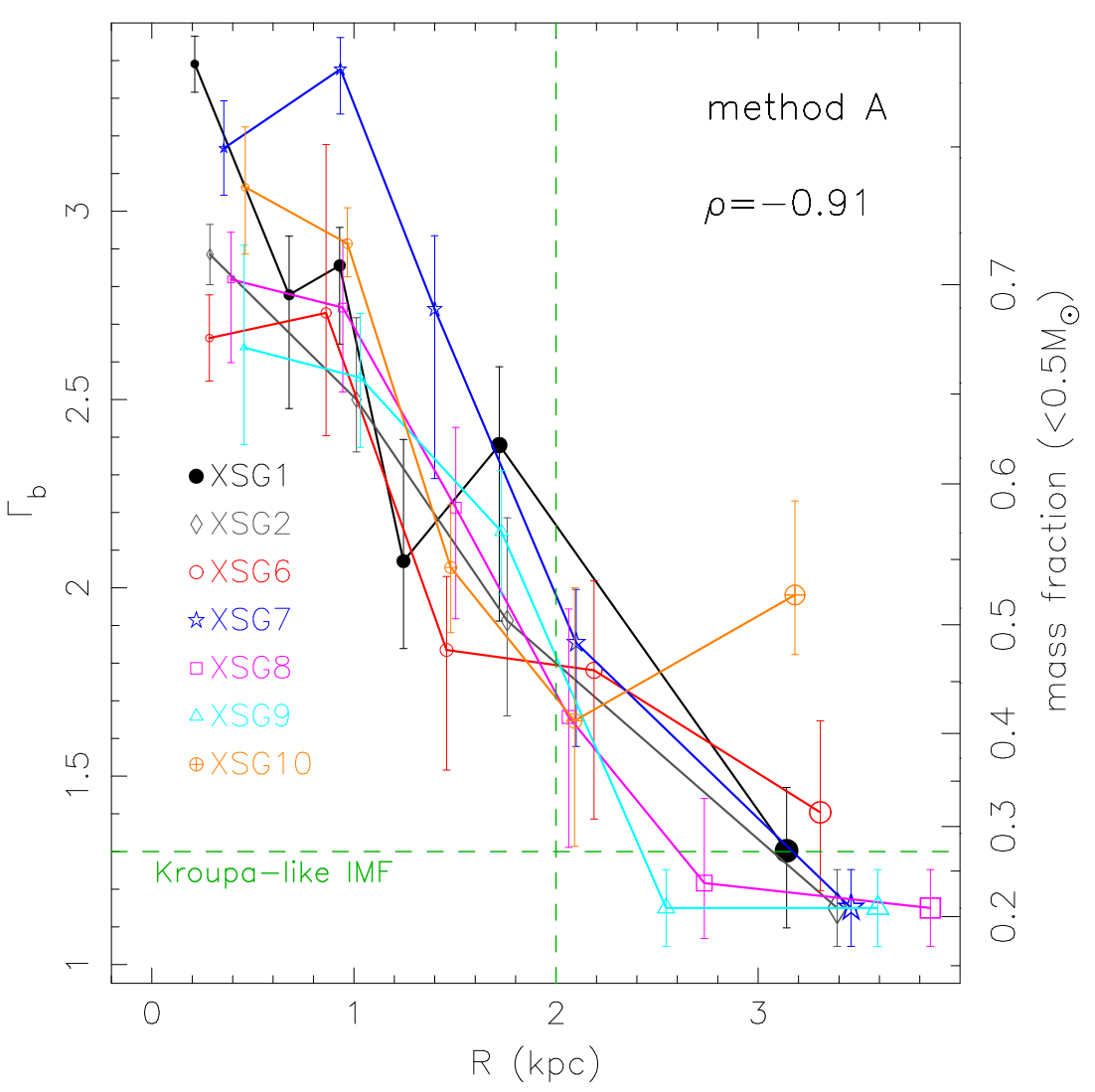

Figure 2. IMF radial gradients in our sample of ETGs, for method A (i.e. including all IMF-sensitive spectral indices in the fitting, and the same stellar population models as in LB16 and LB17; see Tab. 2). The y-axes report the IMF slope $\Gamma_{\mathrm{b}}$ (left) and the mass fraction of low-mass $\left(<\mathrm{M}_{\odot}\right)$ stars in the IMF, as defined in LB13 (see equation 4 of that paper). Different galaxies are plotted with different colors and line types, as labeled. Symbol sizes increase with galactocentric distance. Error bars denote $1 \sigma$ uncertainties. An IMF radial gradient is found for all galaxies, with a Kroupa-like IMF $\left(\Gamma_{\mathrm{b}} \sim 1.3\right.$; see horizontal dashed line) at large radii ( $\left.\gtrsim 2 \mathrm{kpc}\right)$, and a bottom-heavy distribution in the center (leftwards the vertical dashed line). In the upper-right, we report the Pearson's correlation coefficient, $\rho$, of $\Gamma_{\mathrm{b}}$ versus $\mathrm{R}$ for the whole data-set (i.e. all galaxies and radial bins).

radial gradients for their sample of seven ETGs over a range of $\sigma$.

For what concerns radial gradients of total metallicity, $\nabla_{\mathrm{Z}}=\delta[\mathrm{Z} / \mathrm{H}] / \delta(\log \mathrm{R})$, Spolaor et al. (2009) reported a wide range of values at high galaxy mass, ranging from null gradients, to gradients as steep as -0.5 dex per decade in radius (see their figure 2). Using the best-fitting value of $[\mathrm{Z} / \mathrm{H}]$ from our XSG spectra (as shown in panel d of Fig. 3), and performing an orthogonal least-square fit of $[\mathrm{Z} / \mathrm{H}]$ vs. $\log \mathrm{R}$, we obtain $\nabla_{\mathrm{Z}}=-0.26 \pm 0.04,-0.10 \pm 0.05,-0.04 \pm 0.05$, $-0.23 \pm 0.06,-0.14 \pm 0.04,-0.09 \pm 0.06,-0.41 \pm 0.04$, for XSG1, XSG2, XSG6, XSG7, XSG8, XSG9, and XSG10, respectively. These values are consistent with those reported by previous spectroscopic studies (see Martín-Navarro et al. 2018 and references therein).

In the case of $[\mathrm{Na} / \mathrm{Fe}]$, most galaxies have rather flat gradients in the inner regions, that drop down significantly in the outermost bins for XSG2, XSG6, and XSG8. On the other hand, XSG1 ${ }^{12}$ has a midly negative $[\mathrm{Na} / \mathrm{Fe}]$ gradient,

12 Notice that $[\mathrm{Na} / \mathrm{Fe}]$ and other stellar population properties for XSG1 are not exactly the same as those reported in LB16 and LB17, as in the present work we are fitting a different set of IMF-sensitive features with respect to those studies. while XSG7, XSG9, and XSG10 tend to have flat gradients. Notice that we obtain $\mathrm{Na}$ abundance ratios as high as $0.8-$ 0.9 dex for some galaxies (XSG1 and XSG7) in our sample. Such high $\mathrm{Na}$ abundances in ETGs may reflect metallicitydependent yields of $\mathrm{Na}$ from high- and intermediate-mass stars (see LB17 for a detailed discussion), though one should notice that, indeed, XSG1 and XSG7 are not the objects with the highest metallicity in our sample. While other studies (e.g. van Dokkum et al. 2017; Parikh et al. 2018) have reported significantly negative radial gradients of $[\mathrm{Na} / \mathrm{Fe}]$ for ETGs, what matters for the present study is that our results remain virtually the same even when excluding $\mathrm{Na}$ features from the analysis (see Sec. 5.1). Moreover, we notice that the Na-MILES stellar population models adopted in the present work are the only set of models taking the joint effect of $[\mathrm{Na} / \mathrm{Fe}]$ and IMF explicitely into account (see LB17).

\subsubsection{Correlations with $\Gamma_{\mathrm{b}}$}

For each panel of Fig. 4, we report the Pearson's coefficient, $\rho$, of the correlation between $\Gamma_{\mathrm{b}}$ and the corresponding parameter on the $\mathrm{x}$-axis. Symbol size increases with galactocentric distance, R. Notice that each plot in Fig. 4 can be 


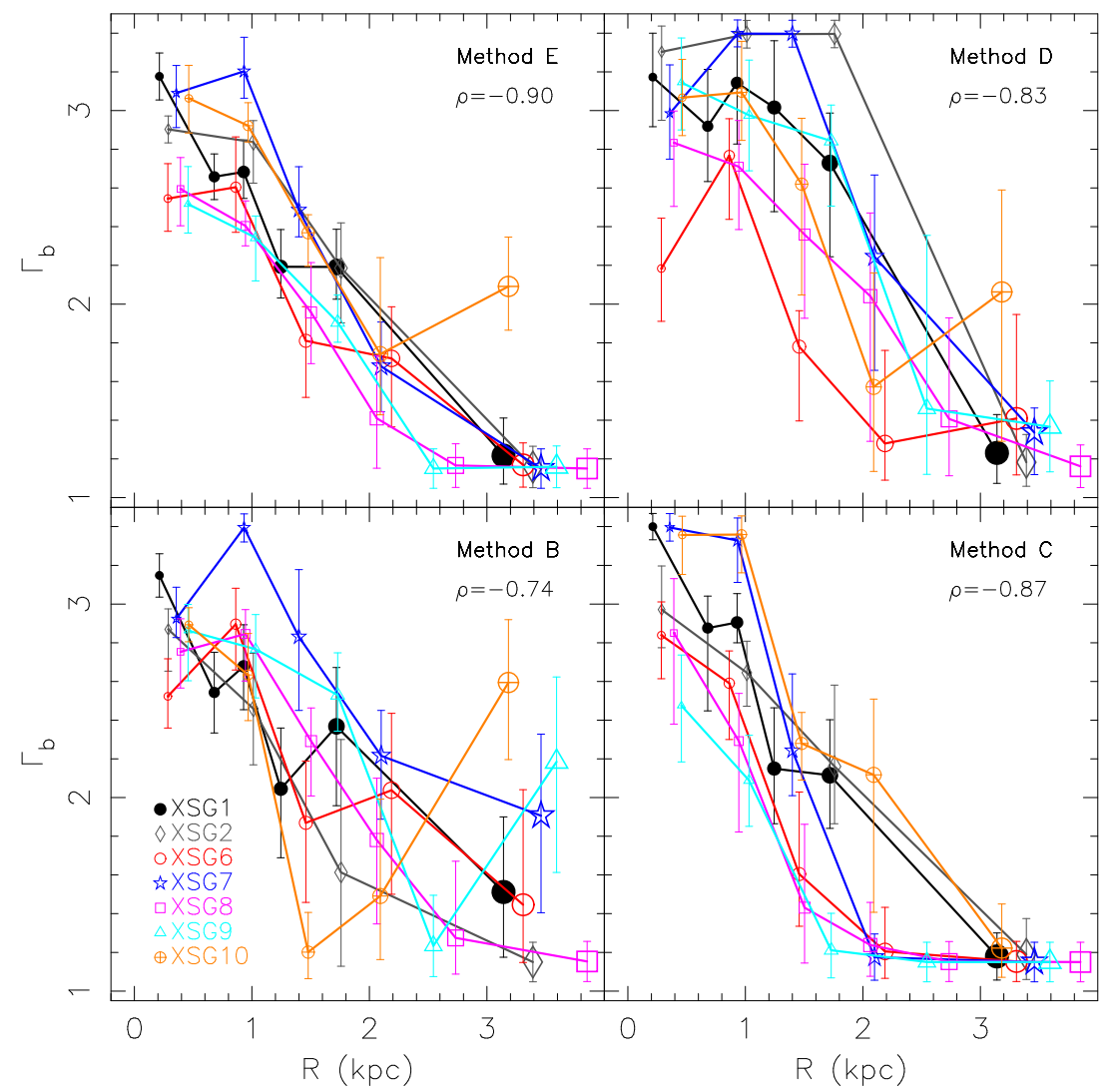

Figure 3. IMF radial gradients for different methods, i.e. different sets of spectral indices and different models (see Tab. 2). For each panel, different galaxies are plotted with different colors and line types (as labeled in the lower-left panel), with symbol sizes increasing with galactocentric distance as in Fig. 2. Upper-left: same as our "reference" method A (i.e. fitting 1SSP models, with all IMF-sensitive indices as well as the total metallicity indicator [MgFe]'; see Fig. 2), but based on models with BaSTI rather than Padova00 isochrones. Upper-right: same as method A, but for 2SSP models, with Balmer lines included in the fitting procedure. Lower-left: same as method A, but excluding $\mathrm{TiO} 1$ and TiO2 $\mathrm{SDSS}$ indices. Lower-right: same as method A, but removing aTiO, Mg4780, and Na indices from the fitting (i.e. using only $[\mathrm{MgFe}]^{\prime}$, TiO1 and $\mathrm{TiO} 2 \mathrm{SDSS}$ ). In the upper-right of each panel, we report the Pearson's correlation coefficient, $\rho$, of $\Gamma_{\mathrm{b}}$ versus $\mathrm{R}$ for the whole data-set (i.e. all galaxies and radial bins). Notice that, for all methods, an IMF radial gradient is detected.

compared directly with the trend of $\Gamma_{\mathrm{b}}$ with galactocentric distance, as shown in Fig. 2. Results can be summarized as follows:

- there is essentially no correlation $(\rho \sim 0)$ of $\Gamma_{\mathrm{b}}$ with age (see panel e of Fig. 4), both individually (i.e. for each single galaxy), and globally (for all data-points);

- correlations with abundance ratios are mild, mainly due to points with lower $[\mathrm{Na} / \mathrm{Fe}]$ and $[\mathrm{Mg} / \mathrm{Fe}]$ in the outermost radial bins (see panels $\mathrm{b}$ and $\mathrm{c}$ of Fig. 4);

- there is a significant correlation of $\Gamma_{\mathrm{b}}$ with velocity dispersion ( $\rho \sim 0.7$; see panel a of Fig. 4 ), with large scatter, in particular for $\sigma \lesssim 300 \mathrm{~km} \mathrm{~s}^{-1}$, where data-points populate a wide region with $1.2 \lesssim \Gamma_{\mathrm{b}} \lesssim 2.5$;

- individual galaxies show a correlation of $\Gamma_{\mathrm{b}}$ with metallicity, but overall, we do not detect a global correlation of IMF slope with $[\mathrm{Z} / \mathrm{H}]$, the correlation coefficient being $\rho \sim 0.24$; in particular, at $[\mathrm{Z} / \mathrm{H}] \sim 0.2$ (where our results are not affected by any extrapolation of the models in the high metallicity regime, see Sec. 3.2), different spectra populate the whole available range of $\Gamma_{\mathrm{b}}$ values, from a bottom-heavy slope (i.e. $\Gamma_{\mathrm{b}} \sim 3$, see the innermost data-points of XSG1 and XSG7), to a Kroupa-like distribution $\left(\Gamma_{\mathrm{b}} \sim 1.3\right.$, see outermost data-points for XSG9 and XSG8);

- galactocentric distance is the quantity giving the tightest correlation to $\Gamma_{\mathrm{b}}(\rho=-0.91$; see Fig. 2 and Sec. 5.1) with respect to all other quantities in Fig. 4.

The lack of correlation of $\Gamma_{\mathrm{b}}$ with $[\mathrm{Z} / \mathrm{H}]$ contrasts with the results of MN15b, where the authors analyzed ETGs in the CALIFA survey, finding that metallicity gives a better correlation to IMF variations with respect to dynamical (e.g. $\sigma$ ) and stellar population properties (e.g. $[\mathrm{Mg} / \mathrm{Fe}]$; see also van Dokkum et al. 2017and Parikh et al. 2018). Panel d in Fig. 4 further illustrates this point, comparing the trend of $\Gamma_{\mathrm{b}}$ with $[\mathrm{Z} / \mathrm{H}]$ for the XSGs, with the locus of $\Gamma_{\mathrm{b}}$ versus $[\mathrm{Z} / \mathrm{H}]$ for CALIFA ETGs (from figure 2 of MN15b), represented as a green shaded area. The XSGs show much steeper (almost vertical) tracks in the $\Gamma_{\mathrm{b}}-[\mathrm{Z} / \mathrm{H}]$ diagram with respect to CALIFA ETGs, covering a wider range of $\Gamma_{b}$ 's at given $[\mathrm{Z} / \mathrm{H}]$ (see above). While this discrepancy might seem surprising, one should notice that the selection criteria of the XSG sample are remarkably different than those of ETGs in MN15b, as (i) the XSGs have been selected to provide a representative sample at the very high-mass end of the 

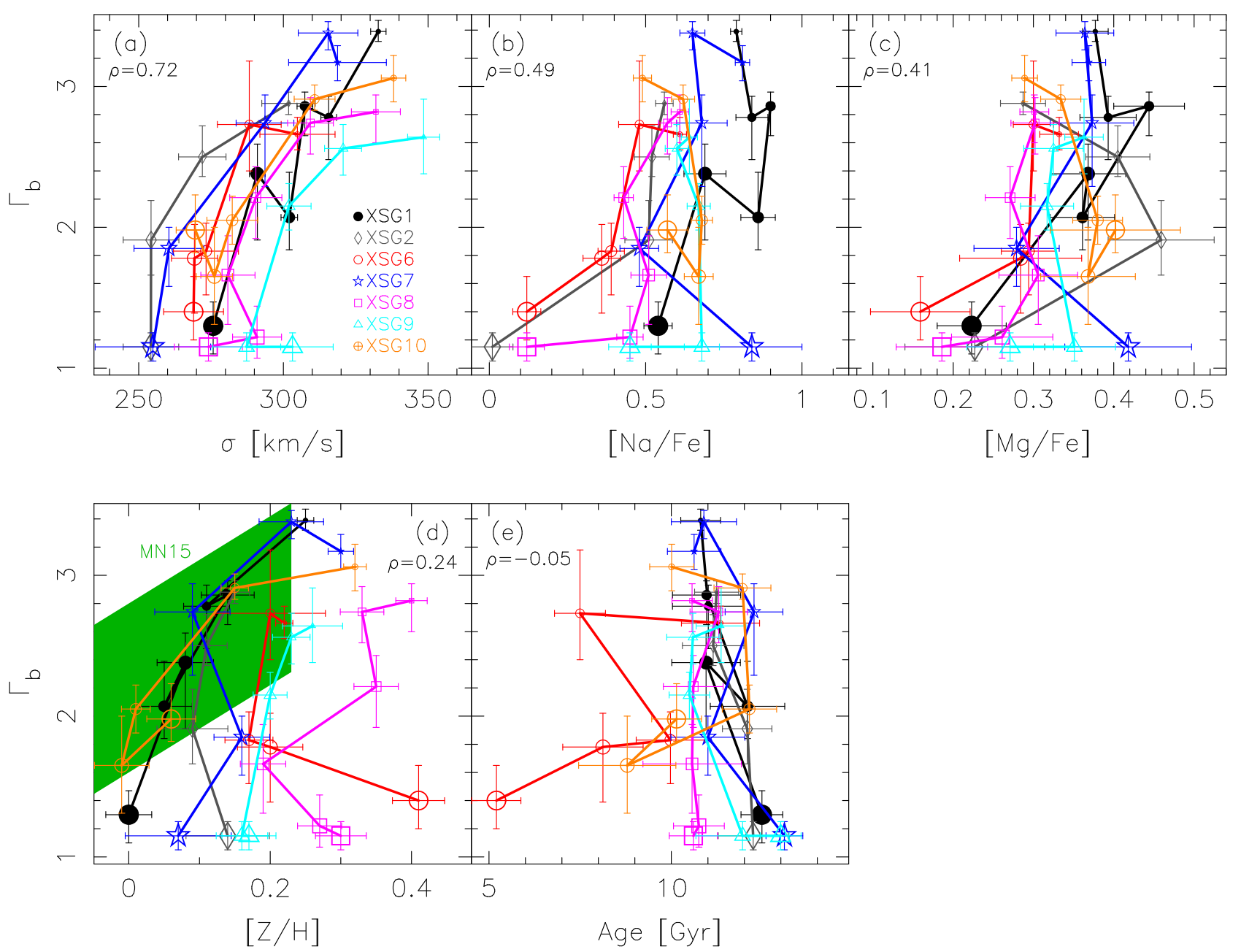

Figure 4. The IMF slope, $\Gamma_{\mathrm{b}}$ is plotted against different quantities, namely velocity dispersion $\sigma$ (panel a), $\mathrm{Na}$ and $\mathrm{Mg}$ abundance ratios $([\mathrm{Na} / \mathrm{Fe}]$ and $[\mathrm{Mg} / \mathrm{Fe}]$; panels b and c, respectively), total metallicity $[\mathrm{Z} / \mathrm{H}](\mathrm{d})$, and age $(\mathrm{e})$. In each panel, we report the value of the Pearson's correlation coefficient, $\rho$, of $\Gamma_{\mathrm{b}}$ versus each quantity, for all data-points (i.e. all galaxies/radial bins). The values of $\Gamma_{\mathrm{b}}$ are for our reference method A (see Tab. 2). Symbols, line types, and error bars follow the same coding as in Figs. 2 and 3 (see also labels in the lower-right corner of panel a). Notice that all correlations are weaker than that with galactocentric distance $(\rho \sim 0.9$; see Fig. 2). The green shaded area in the bottom-left panel marks the locus of the IMF- $[\mathrm{Z} / \mathrm{H}]$ relation from MN15b (see the text).

galaxy population (see Sec. 2); and (ii) none of the CALIFA ETGs in MN15b have central velocity dispersion larger than $300 \mathrm{kms}^{-1}$ (see fig. 1 of MN15b), unlike the XSGs; (iii) as a consequence of point (ii), ETGs in CALIFA populate a $[\mathrm{Z} / \mathrm{H}]$ range from about -0.3 up to $[\mathrm{Z} / \mathrm{H}] \sim+0.2$, while all XSG spectra are either in the solar or super-solar metallicity regime (see panel d of Fig. 4). Indeed, ETGs at very highmass end might have different radial IMF gradients than the average population of ETGs - depending on the way they formed and accreted their outer regions - hence explaining the difference between our findings and those in MN15b (as well as some other studies of IMF radial gradients). We further discuss this point in Sec. 6 .

As shown in panel e of Fig. 4, we do not find any correlation of $\Gamma_{\mathrm{b}}$ with age. Although we do not expect any significant contribution of young stellar populations in our sample of massive ETGs, we can investigate this point further, by looking for spectra with emission lines (if any) in the X-Shooter sample. As shown in App. F, some of our galax- ies, i.e. XSG1, XSG6, and XSG8, show signs of emission lines in the optical spectral range. The analysis of diagnostic diagrams shows that this emission implies a ionization pattern typical of retired stellar populations (i.e. emission lines produced by hot post-asymptotic giant branch stars) in the galaxy central regions (see the Appendix for detail). For these objects, one may expect that 1SSP and 2SSP models might not give an accurate description of the galaxy starformation history, possibly affecting our results. However, as shown in Fig. 4, XSG1, XSG6, and XSG8 do not show different IMF profiles, neither they exhibit peculiar abundance patterns, compared to other galaxies. In other terms, the presence of (weak) emission in massive ETGs does not affect at all the IMF variations.

We also notice that the IMF profiles of the XSGs do not depend on environment. Satellite galaxies in our sample (XSG2 and XSG7) have IMF profiles similar to those for the rest of the sample, although XSG7 is the galaxy with bottom-heaviest IMF in the three innermost radial bins 


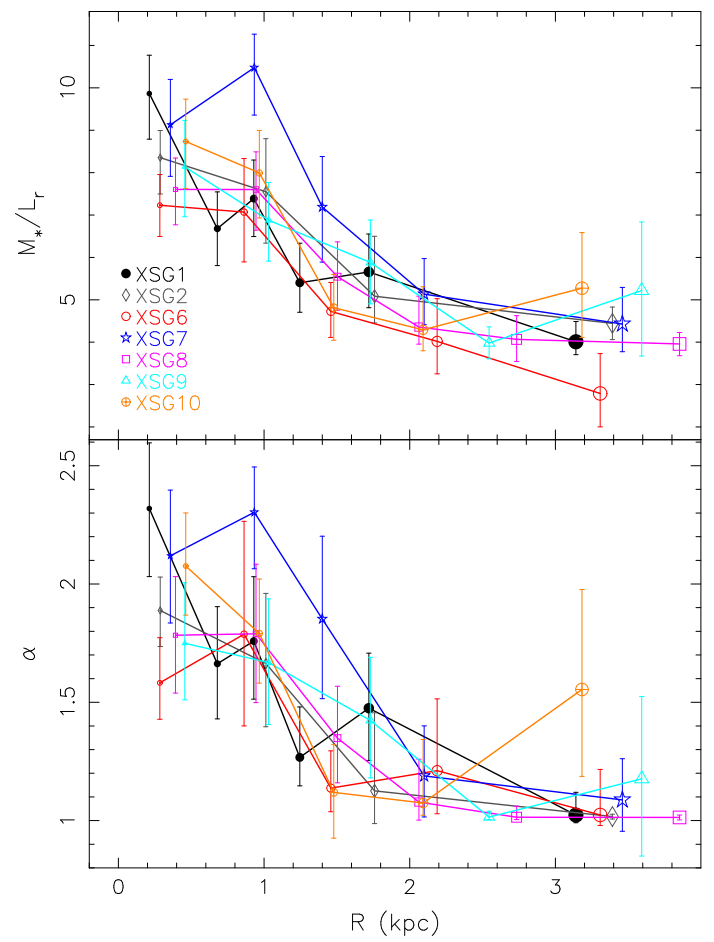

Figure 5. Radial gradients of the r-band stellar mass-to-light ratio, $\mathrm{M}_{\star} / \mathrm{L}_{\mathrm{r}}$ (upper panel), and the "mismatch parameter" $\alpha$ (lower panel). The $\alpha$ is given by the estimated $M_{\star} / L_{r}$ normalized to that for a Kroupa-like IMF (assuming the same age and metallicity). Error bars correspond to $1 \sigma$ uncertainties. In order to account for systematics in the $\mathrm{M} / \mathrm{L}$ estimates, both $\mathrm{M}_{\star} / \mathrm{L}_{\mathrm{r}}$ and $\alpha$ are obtained by averaging out results from different methods (i.e. methods A, B, and E; see the text and Tab. 2). Different galaxies are plotted with different symbols (whose size increases with galactocentric distance) and colours (see labels in the upper panel), with the same coding as in Fig. 2.

among all the XSGs (see Fig. 2). However, as detailed in App. A, the hierarchy of XSG7 is uncertain; moreover, both XSG2 and XSG7 might have been actually centrals 2-3 Gyr before infalling into their current parent groups. In other words, all the XSGs might have spent most of their evolutionary history as group centrals. Notice that the lack of correlation between IMF profiles and galaxy environment is consistent with the results of Rosani et al. (2018), who found that the IMF- $\sigma$ relation is independent of hierarchy.

\section{$5.3 \mathrm{M} / \mathrm{L}$ radial gradients}

Fig. 2 shows that all the XSGs exhibit a bottom-heavy IMF, i.e. an excess of low-mass stars, in the galaxy central regions. Since low-mass stars dominate the mass budget of a stellar population, Fig. 2 implies, in turn, a significant radial gradient in the stellar mass-to-light ratio. Fig. 5 (top panel) shows the expected r-band mass-to-light ratio, $M_{\star} / L_{r}$, for all the XSGs, as a function of galactocentric distance. Since the variation of IMF slope (with respect to that of other parameters, such as $[\mathrm{Z} / \mathrm{H}])$ dominates the $\mathrm{M}_{\star} / \mathrm{L}_{\mathrm{r}}$, the top panel of Fig. 5 looks similar to Fig. 2, i.e. all galaxies show a similar $\mathrm{M}_{\star} / \mathrm{L}_{\mathrm{r}}$ radial gradient, with $6 \lesssim \mathrm{M}_{\star} / \mathrm{L}_{\mathrm{r}} \lesssim 11$ in the center, down to $\mathrm{M}_{\star} / \mathrm{L}_{\mathrm{r}} \sim 4$ in the outermost radial bins, the

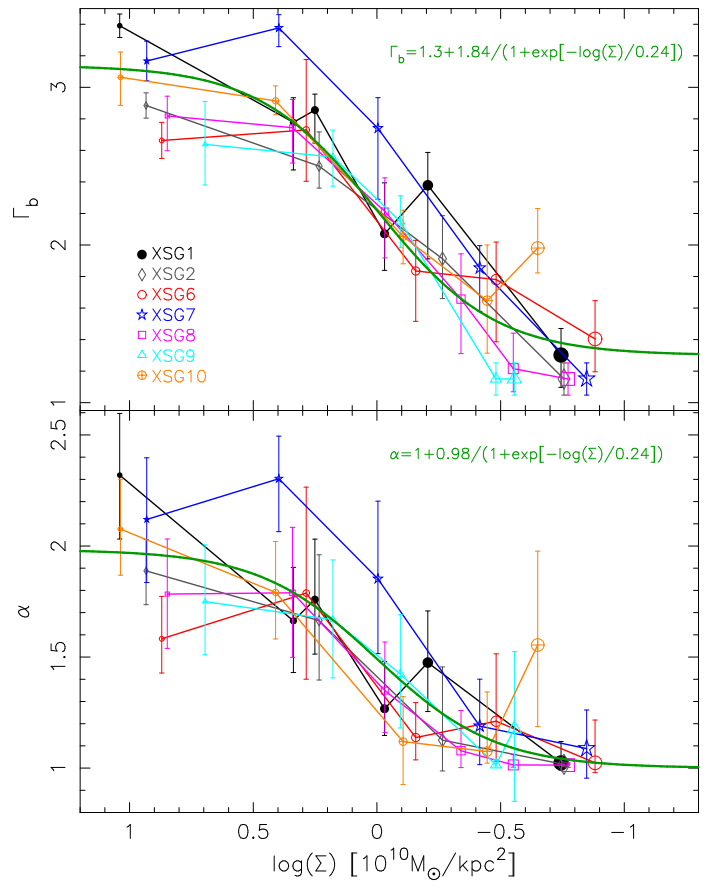

Figure 6. IMF slope $\Gamma_{\mathrm{b}}$ (top) and mismatch parameter $\alpha$ (bottom) are plotted as a function of logarithmic projected stellar mass density $\Sigma$, for our reference fitting scheme (method A; see Tab. 2). Different galaxies are plotted with different symbols (with symbol size increasing with galactocentric distance) and colours (see labels in the upper panel), with the same coding as in Fig. 2. Green curves are the best-fitting functional forms (whose equations are given in the upper-right of each panel) that describe the trend for all galaxies in our sample (see the text).

latter value corresponding, approximately, to the M/L expected for a Milky Way-like IMF.

The $\mathrm{M} / \mathrm{L}$ radial gradients are further shown in the bottom panel of Fig. 2, where we plot the "mass-excess" parameter, $\alpha$, in $\mathrm{r}$ band, as a function of galactocentric distance. The $\alpha$ is defined as the actual stellar mass-to-light ratio of a stellar population, normalized to that predicted for a Kroupa-like IMF, assuming the same parameters (e.g. age and metallicity) as the given population. The $\alpha$ parameter has been first introduced by CvD12a to single out the effect of variations in the IMF with respect to that of other parameters. For a Kroupa-like IMF, one has $\alpha=1$. In the innermost bins, the XSGs have $\alpha$ significantly above one, with values between $\sim 1.7$ (e.g. XSG6 and XSG9) and $\sim 2.2$ (e.g. XSG1 and XSG7), while at larger distances ( $>2 \mathrm{kpc}$ ), we find $\alpha \sim 1$ (i.e. a Milky Way-like distribution), consistent with Fig. 2.

However, we warn the reader about a "swift" use of results in Fig. 5. In fact, both the mass-excess factor and the stellar M/L depend significantly on the assumed IMF functional form (see LB13 and LB16). For instance, assuming a slightly higher low-mass end cutoff than that used in our models (i.e. $0.1 M_{\odot}$; see Sec. 3), one could decrease the stellar M/L, without changing significantly the predicted line-strength of IMF-sensitive spectral indices (see, e.g., Barnabé et al. 2013), as most features are actually insensi- 
tive to very low-mass stars ${ }^{13}$. On the other hand, as shown in Lyubenova et al. (2016) (see also LB16), the (bimodal) IMF functional form provides consistent M/L estimates with respect to dynamical studies. We plan to come back to this issue in a forthcoming paper, comparing dynamical and stellar population $\mathrm{M} / \mathrm{L}$ estimates for the XSGs.

The presence of steep M/L gradients in our sample of massive ETGs, implies that luminosity-weighted M/L ratios within a circular aperture depend significantly on the aperture size. In particular, within a region of $1 \mathrm{R}_{\mathrm{e}, \mathrm{T}}$ (the effective radius from the $\mathrm{B}+\mathrm{D}$ decomposition, see Sec. 3 ), our IMF profiles imply luminosity-weighted values of $\alpha$ in the range from $1.15 \pm 0.05$ (for XSG8) to $1.6 \pm 0.1$ (for XSG7), with an average of $\alpha=1.3 \pm 0.1$. In other terms, under the IMF parametrization adopted in the present work, massive ETGs should have M/L's inside $1 \mathrm{R}_{\mathrm{e}}$, only $\sim 30 \%$ larger on average with respect to the case of a radially constant Milky Way-like IMF. Notice that (i) to perform these estimates of $\alpha$, we have extrapolated the M/L profiles outside the radial range probed by $\mathrm{X}$-Shooter, assuming that the IMF remains consistent with a Milky Way-like distribution ( $\alpha=1$ ) outwards; and (ii) the luminosity-weighted $\alpha$ within $\mathrm{R}_{\mathrm{e}}$ would be even lower when considering effective radii from Sérsic models $\left(\mathrm{R}_{\mathrm{e}, \mathrm{S}}\right)$, as some galaxies (XSG6, XSG7, XSG8, XSG9) have $R_{e, S}$ significantly larger than $R_{e, T}$ (Tab. C1).

Our M/L estimates can be compared to those of Smith, Lucey \& Conroy (2015, hereafter SLC15), who found three massive ETGs (named SNL-0, SNL-1, and SNL-2) having spectroscopic signatures consistent with a bottomheavy IMF, but nevertheless a "light" M/L, as implied by lensing/dynamical constraints. Within a typical region of $\sim 2 \mathrm{kpc}$, the SNL's have $\alpha=1.06 \pm 0.1\left(\alpha=1.3 \pm 0.13^{14}\right)$ in the case where dark-matter (no dark-matter) contribution, within the galaxy central regions, is taken into account (see table 1 of Newman et al. 2017). On the other hand, within a region of $2 \mathrm{kpc}$, for the XSGs, we estimate a median luminosity-weighted (circularized) $\alpha$ of $\sim$ $1.5 \pm 0.15$, which is consistent with the SNLs only in the case of no dark-matter. Increasing the low-mass end cutoff in the IMF, would likely match better the M/L of SNL ETGs (Newman et al. 2017). However, this requires a somewhat fine-tuned scenario, whereby the IMF has an excess of low-mass stars (to match the spectroscopic signatures), but lacks the very low-mass stars (to match the M/L).

13 An exception is the Wing-Ford band, FeH0.99, at $\lambda \sim 9915 \AA$, whose sensitivity peaks in the very low-mass range, below $\sim$ $0.3 M_{\odot}$ (see CvD12a). As shown in LB16, the bimodal IMF is able to match the FeH0.99 measured in our spectra for XSG1, in contrast to a single power-law parametrization, predicting too high FeH0.99. However, FeH0.99 is also strongly dependent on $[\mathrm{Mg} / \mathrm{Fe}]$ and $[\mathrm{Na} / \mathrm{Fe}]$, and the $\mathrm{FeH}$ molecule is not implemented yet in the Na-MILES models (see LB17), hampering the interpretation of this feature (see, e.g., van Dokkum et al. 2017; Vaughan et al. 2018).

14 These values of $\alpha$, and their errors, are computed by averaging values for SNL-0, SNL-1, and SNL-2 from table 1 of Newman et al. (2017).

\subsection{IMF variations and mass density}

Since all our targets have similar values of the central velocity dispersion (which is a proxy of galaxy mass, given the small rotation velocity of the XSGs), the presence of IMF radial gradients in our sample suggests a correlation of IMF slope with mass density. Fig. 6 shows the trend with surface mass density $\Sigma$, for both IMF slope, $\Gamma_{\mathrm{b}}$ (top panel), and the "mass-excess" factor, $\alpha$, i.e. the mass-to-light ratio normalized to that for a Kroupa-like IMF (bottom-panel). Indeed, a significant correlation exists ${ }^{15}$, that can be modeled with the analytic functions shown in Fig. 6 (see green solid curves and equations), having a plateau at both high and low density (corresponding to a bottom-heavy and Kroupa-like IMF, respectively), and a transition region at $\Sigma \sim 10^{10} \mathrm{M}_{\odot} \mathrm{kpc}^{-2}$. This characteristic density corresponds to a characteristic scale of $\sim 1-2 \mathrm{kpc}$, as in Fig. 2. However, we caution the readers that the high-density plateau in Fig. 6 is (partly) driven by the IMF determination for the central radial bins of the XSGs, where some extrapolation of stellar population models to the high-metallicity regime is required (see Sec. 3).

The correlation in Fig. 6 may appear in disagreement with the result of Spiniello et al. (2015, hereafter SBK15), who claimed an anticorrelation of IMF slope and galaxy mass density. We notice, however, that the finding of SBK15 refers to the IMF slope in the central galaxy regions versus total mass density (the latter estimated as $\sigma^{2} / R_{\mathrm{e}}^{2}$ ), while in Fig. 6 we show a local correlation of IMF slope and (stellar) mass density. Since all the XSGs have a similar $\sigma_{0}$ (especially when considering the central circular aperture probed by the SDSS fiber, see Tab. 1), "total" mass density in our sample is inversely proportional to $\mathrm{R}_{\mathrm{e}}^{2}$. Indeed, although the galaxies with largest $R_{e}$ in our sample (i.e. XSG6, XSG8, and XSG9) tend to have a lower IMF in the central radial bins (Fig. 2) than the others, the IMF in their center is still bottom-heavy $\left(\Gamma_{\mathrm{b}} \sim 2.75^{16}\right)$, and overall, their IMF radial gradients are similar to those for the other XSGs ${ }^{17}$ (Fig. 2). Therefore, our data do not point to a fundamental anticorrelation of IMF slope and total density (as in SBK15), but rather, a significant correlation of local IMF slope and projected mass density.

Interestingly, Barone et al. (2018) have recently found a correlation of stellar population properties, such as age and $[\mathrm{Mg} / \mathrm{Fe}]$, with surface mass density, interpreting these trends as a result of compactness-driven quenching mechanisms, and/or as a consequence of star-formation rate (SFR)-gas density relation in the disk-dominated progenitors of ETGs. We notice that our sample of ETGs does not exhibit significant age and $[\mathrm{Mg} / \mathrm{Fe}]$ radial gradients (see Sec. 5.2.1), but

15 Notice that the computation of $\Sigma$ involves the actual $M * / L$, that, in turn, depends on $\Gamma_{\mathrm{b}}$. In other terms, by construction, the variables on the $\mathrm{x}$ - and $\mathrm{y}$-axes of Fig. 6 are not independent. However, the relation in Fig. 6 is a genuine one. In fact, we found that the correlation of $\Gamma_{\mathrm{b}}$ with luminosity (rather than mass) density is very similar to that of $\Gamma_{\mathrm{b}}$ versus $\Sigma$.

16 This is the average of $\Gamma_{\mathrm{b}}$ values at $R \sim 0$ for XSG6, XSG8, and XSG9, when considering our reference fitting scheme.

17 In fact, computing the luminosity-weighted value of $\alpha$ for XSG6, XSG8, and XSG9, within a mock circular aperture of radius 1", gives $1.74 \pm 0.04$, which is fully consistent with the average value of $1.94 \pm 0.2$ for the other XSGs. 
nevertheless the IMF slope correlates with surface-mass density. Therefore, IMF variations in our sample do not seem to have necessarely the same origin as the stellar population trends found by Barone et al. (2018).

\section{DISCUSSION}

All massive ETGs in our sample (XSGs) show very similar, steep, IMF radial gradients, over a physical region of $\sim 4 \mathrm{kpc}$ from the galaxy centers. The IMF changes from bottom-heavy, to Kroupa-like, at a characteristic distance of $\sim 2 \mathrm{kpc}$ (Fig. 2), or, alternatively, at a typical surface mass density of $\sim 10^{10} M_{\odot} k p c^{-2}$ (Fig. 6). In a twophase model of galaxy formation, the core of massive galaxies is expected to form "in-situ" at high redshift through a rapid dissipative process of star formation, while the outer galaxy envelopes are expected to build up at later epochs through major/minor mergers (Oser et al. 2010, 2012; Navarro-González et al. 2013). This might be the case also for massive BCGs (Laporte et al. 2013; Oogi et al. 2016) - an aspect that is relevant for the present work, as most of our targets are actually group centrals, or might have been centrals before accretion onto a galaxy group a few Gyrs before the epoch of observation (see App. A). The characteristic scale of $\sim 2 \mathrm{kpc}$ for IMF gradients in our sample coincides with the typical size of high-redshift massive compact galaxies (the so-called "red nuggets"; see, e.g., Trujillo et al. 2007; Damjanov et al. 2011; Saracco, Longhetti, Gargiulo 2011). This suggests that our IMF gradients are connected to those of massive compact galaxies. Indeed, a few massive compact galaxies have been found also at $\mathrm{z} \sim 0$, the prototype of these objects being NGC 1277 (Trujillo et al. 2014). These galaxies, that are believed to be the "relics" of the high-z red nuggets, have significant rotation and velocity dispersion gradients, with shallow IMF radial gradients (the IMF being bottom-heavy) over a region of at least 3-4 kpc (Martín-Navarro et al. 2015c; Ferré-Mateu et al. 2017). More in detail, Ferré-Mateu et al. (2017) found that at a galactocentric distance of $R \sim 3 \mathrm{kpc}$ (corresponding to a mass density of $\sim 10^{9} \mathrm{M}_{\odot} / \mathrm{kpc}^{2}$ ), the IMF slope of relic galaxies is $\Gamma_{\mathrm{b}} \gtrsim 2.5$ (see their figure 5), which is inconsistent with the trends in Figs. 2 and 6 . Moreover, in Martín-Navarro et al. (2018), we found that relic galaxies tend to have $[\mathrm{Mg} / \mathrm{Fe}]$ profiles that increase outwards, while normal ETGs, as well as the XSGs (see Fig. 4), tend to have either flat, or negative, $[\mathrm{Mg} / \mathrm{Fe}]$ radial gradients. Therefore, either the central regions of massive $\left(\sigma>300 \mathrm{~km} \mathrm{~s}^{-1}\right)$ ETGs formed "in-situ" by a different channel than massive relic galaxies at $z \sim 0$, or other processes, such as merging/accretion affected significantly the region from $\sim 2$ to $4 \mathrm{kpc}$, producing the steep IMF gradients we observe in our sample. Dry merging would unlikely explain, by itself, our observations. In MN15b, we found a tight correlation of local metallicity and IMF slope for intermediate-mass ETGs, as probed by the CALIFA survey (see also Parikh et al. 2018). Therefore, any accreted material in massive ETGs would likely follow the CALIFA IMF$[\mathrm{Z} / \mathrm{H}]$ relation, while as noted in Sec. 5.2, at fixed metallicity we find a wide range of IMF slopes in our sample. In particular, at super-solar metallicity $([\mathrm{Z} / \mathrm{H}] \sim 0.2)$, we find IMF slopes ranging from a bottom-heavy to a Kroupa-like distri- bution among different XSGs. As discussed in Weidner et al. (2013) and Ferreras et al. (2015), in order to reconcile an excess of low-mass stars with the high metallicity content of massive galaxies, one may invoke a scenario whereby star formation starts with a top-heavy phase, switching to a bottom-heavy distribution at later times. Our results imply that the regions corresponding to a few kpc in very massive galaxies may have formed/evolved through dissipative processes (e.g. wet mergers at high z), whose physical conditions were different than those in high-redshift compact cores, hence preventing an efficient switch from a top to bottom-heavy phase at all radii (see Vazdekis et al. 1997).

We point out that the present work is the first attempt to perform a systematic study of IMF variations in very massive $\left(\sigma>300 \mathrm{~km} \mathrm{~s}^{-1}\right)$ ETGs. Sarzi et al. (2018) derived the IMF radial profile for M87 (the giant elliptical galaxy at the center of the Virgo cluster), finding a consistent profile to that for XSG1. Vaughan et al. (2018) also derived spatially resolved measurements of the stellar IMF in NGC 1399, the largest elliptical galaxy in the Fornax Cluster. They found a super-Salpeter IMF out to $\sim 0.7 \mathrm{R}_{\mathrm{e}}$, with a distribution marginally consistent to a Milky-Way IMF beyond $R_{e}$, corresponding to a characteristic scale of $\sim 3-$ $4 \mathrm{kpc}$. This scale is significantly larger than that for the XSGs and M87, meaning that a wider range of IMF profiles might exist, than those for our XSG sample. This might also be the case for SNL-0 (Smith, Lucey \& Conroy 2015; Newman et al. 2017), a very massive $\left(\sigma>300 \mathrm{~km} \mathrm{~s}^{-1}\right)$ ETG whose lensing-based IMF normalization is consistent with a Milky-Way distribution within a region of $\sim 1.9 \mathrm{kpc}$. We notice that M87 has a rather flat velocity dispersion profile, with little rotation (less than $\sim 20 \mathrm{~km} \mathrm{~s}^{-1}$ within the region probed by Sarzi et al. 2018), as it is also the case for the XSGs. On the contrary, NGC 1399, as well as compact massive ETGs, have significant velocity dispersion and rotation gradients. Therefore, we speculate that there might exist some anti-correlation of IMF and kinematics radial gradients, with galaxies showing shallower kinematics profiles having steeper IMF gradients. Qualitatively, such anticorrelation might result from the general properties of the Jeans mass, $\mathrm{M}_{\mathrm{J}} \propto \mathrm{T}^{3 / 2} \rho^{-1 / 2}$, with $\mathrm{T}$ and $\rho$ being temperature and density, respectively. Assuming that local velocity dispersion $(\sigma)$ is a proxy for $\mathrm{T}$, and given the shallow $\sigma$ gradients of the XSGs, one would have $\mathrm{M}_{\mathrm{J}} \propto \rho^{-1 / 2}$, i.e. the correlation of IMF and mass density in Fig. 6 would be a consequence of the dependence of $\mathrm{M}_{\mathrm{J}}$ on local density. On the contrary, in systems with a stronger $\sigma$ drop with radius (such as the relic galaxies), the decrease of both $\mathrm{T}$ and $\rho$ with radius would imply a more constant $\mathrm{M}_{\mathrm{J}}$, i.e. shallower IMF radial gradients (as observed).

Overall, our results imply that there seems to be no single driver of IMF variations in early-type galaxies, and in particular, that metallicity cannot be the only culprit of IMF variations in stellar systems. A similar conclusion has been drawn by Villaume et al. (2017), who analyzed IMF variations in compact, low velocity dispersion, stellar systems within a wide metallicity range, finding smaller IMF variations than in ETGs. Martín-Navarro et al. (2019) also found that the two-dimensional map of IMF variations in a lenticular galaxy does not resemble its metallicity (2D) distribution. Instead, in our sample of massive ETGs, we find a clear correlation of IMF slope with local mass density, as 
well as galactocentric distance in physical units. These results, together with previous findings on IMF radial gradients, show that IMF variations in galaxies likely result from the complex formation processes and mass accretion history of galaxies at different galactocentric distances, showing the importance of determining IMF gradients to constrain the galaxy formation scenario.

\section{ACKNOWLEDGEMENTS}

Based on observations made with ESO Telescopes at the Paranal Observatory under programmes ID 092.B0378, 094.B-0747, 097.B-0229 (PI: FLB). We thank the anonymous referee for the helpful comments and suggestions. FLB acknowledges the Instituto de Astrofísica de Canarias for the kind hospitality when this project started. We thank dr. J. Alcalá for the insightful discussions and help with the reduction of X-Shooter spectra. We have made extensive use of the SDSS database (http://www.sdss.org/collaboration/credits.html). FLB, AV, JFB acknowledge support from grant AYA201677237-C3-1-P from the Spanish Ministry of Economy and Competitiveness (MINECO). GvdV acknowledges funding from the European Research Council (ERC) under the European Union's Horizon 2020 research and innovation programme under grant agreement No 724857 (Consolidator Grant ArcheoDyn). FLB also acknowledges financial support from the European Union's Horizon 2020 research and innovation programme under the Marie Sklodowska-Curie grant agreement n.721463 to the SUNDIAL ITN network.

\section{REFERENCES}

Abazajian, K. N., Adelman-McCarthy, J. K., Agueros, M. A., et al., 2009, ApJS, 182, 534

Alton, P. D., Smith, R. J., Lucey, J. R., 2017, MNRAS, 468, 1594

Alton, P. D., Smith, R. J., Lucey, J. R., 2018, MNRAS, 478, 4464

Baldwin, J. A., Phillips, M. M., Terlevich, R., 1981, PASP, 93, 5

Barnabé, M., Spiniello, C., Koopmans, L. V. E., Trager, S. C., Czoske, O., Treu, T., 2013, MNRAS, 436, 253

Barone, T. M., et al., 2018, ApJ, 856, 64

Bastian N., Covey K. R., Meyer M. R., 2010, ARA\&A, 48, 339

Bate M. R., Bonnell I. A., 2005, MNRAS, 356, 1201

Bell E. F., McIntosh D. H., Katz N., Weinberg M. D., 2003, ApJS, 149,289

Bertelli Motta, C., Clark, P. C., Glover, S. C. O., Klessen, R. S., Pasquali, A., 2016, MNRAS, 462, 4171

Blanton, M. R., Schlegel, D. J., Strauss, M. A., et al., 2005, AJ, 129,2562

Bruzual, G., Charlot, S., 2003, MNRAS, 344, 1000

Cappellari, M., Emsellem, E., 2004, PASP, 116, 138

Cappellari M., et al., 2012, Natur, 484, 485

Cappellari M., et al., 2013, MNRAS, 432, 1862

Cassisi, S., Castellani, V., Ciarcelluti, P., Piotto, G., Zoccali, M., 2000, MNRAS, 315, 679

Cassisi, S., Salaris, M., Castelli, F., Pietrinferni, A., 2004, ApJ, 616, 498

Cenarro, A. J., Cardiel, N., Gorgas, J., Peletier, R. F., Vazdekis, A., Prada, F., 2001a, MNRAS, 326, 959

Cenarro, A. J., Gorgas, J., Vazdekis, A., Cardiel, N., Peletier, R. F., 2003, MNRAS, 339, L12

Cervantes, J. L., Vazdekis, A., 2009, MNRAS, 392, 691

Chabrier G., Hennebelle P., Charlot S., 2014, ApJ, 796, 75
Cid Fernandes, R., Stasińska, G., Mateus, A., Vale Asari, N., Chieffi, A., Limongi, M., 2004, ApJ, 608, 405

Clauwens B., Schaye J., Franx M., 2016, MNRAS, 462, 2832

Conroy, C., van Dokkum, P., 2012a, ApJ, 747, 69 (CvD12a)

Conroy, C., van Dokkum, P., 2012b, ApJ, 760, 71

Cushing, M. C., Rayner, J. T., Vacca, W. D., 2005, ApJ, 623, 1115

Daddi, E., et al., 2005, ApJ, 626, 680

Damjanov, I., et al., 2011, ApJL, 739, 44

Davé, R., 2008, MNRAS, 385, 147

de La Rosa I. G., La Barbera F., Ferreras I., de Carvalho R. R., 2011, MNRAS, 418, L74

Faber S. M., French H. B., 1980, ApJ, 235, 405

Ferré-Mateu, A., Trujillo, I., Martín-Navarro, I., Vazdekis, A., Mezcua, M., Balcells, M., Dom'inguez, L, 2017, MNRAS, 467, $1929 \mathrm{~F}$

Ferreras, I., La Barbera, F., de la Rosa, I. G., Vazdekis, A., de Carvalho, R. R., Falcón-Barroso, J., Ricciardelli, E., 2013, MNRAS, 429, L15 (F13)

Ferreras, I., Weidner, C., Vazdekis, A., La Barbera, F., 2015, MNRAS, 448, 82

Fontanot F., La Barbera F., De Lucia G., Pasquali A., Vazdekis A., 2018, MNRAS, 479, 5678

Geha M., et al., 2013, ApJ, 771, 29

Girardi, L., Bressan, A., Bertelli, G., Chiosi, C., 2000, A\&AS, 141,371

Greene, J. E., Janish, R., Ma, C.-P., McConnell, N. J., Blakeslee, J. P., Thomas, J., Murphy, J., 2015, ApJ, 807, 11

Grevesse, N., Sauval, A. J., 1998, SSrv, 85, 161

Gunawardhana M. L. P., et al., 2011, MNRAS, 415, 1647

Gutcke T. A., Springel V., 2019, MNRAS, 482, 118

Hopkins P. F., 2013, MNRAS, 433, 170

Hopkins A. M., 2018, PASA, 35, 39

Jeong, H., Yi, S. K., Kyeong, J., Sarzi, M., Sung, E.-C., Oh, K., 2013, ApJS, 208, 7J

Jeřábková T., Hasani Zonoozi A., Kroupa P., Beccari G., Yan Z., Vazdekis A., Zhang Z.-Y., 2018, A\&A, 620, A39

Kroupa P., 2001, MNRAS, 322, 231

Krumholz M. R., Myers A. T., Klein R. I., McKee C. F., 2016, MNRAS, 460, 3272

Kroupa, P., 2001, MNRAS, 322, 231

La Barbera, F., et al. 2008, PASP, 120, 681

La Barbera, F., de Carvalho, R. R., de la Rosa, I. G., Lopes, P. A. A., Kohl-Moreira, J. L., Capelato, H. V., 2010a, MNRAS, 408, 1313 (Paper I)

La Barbera, F., Ferreras, I., Vazdekis, A., de la Rosa, I. G., de Carvalho, R. R., Trevisan, M., Falcón-Barroso, J., Ricciardelli, E., 2013, MNRAS, 433, 3017 (LB13)

La Barbera F., Ferreras I., Vazdekis A., 2015, MNRAS, 449, L137

La Barbera F., Vazdekis A., Ferreras I., Pasquali A., Cappellari M., Martín-Navarro I., Schönebeck F., Falcón-Barroso J., 2016, MNRAS, 457, 1468 (LB16)

La Barbera F., Vazdekis A., Ferreras I., Pasquali A., Allende Prieto, C., Rc̈k, B., Aguado, D. S., Peletier, R. F., 2017, MNRAS, 464, 3597 (LB17)

Laporte, F. P. L., White, S. D. M., Naab, T., Ostriker, Gao, L., 2013, MNRAS, 435, 901

Leier D., Ferreras I., Saha P., Charlot S., Bruzual G., La Barbera F., 2016, MNRAS, 459, 3677

Lyubenova, M., Martín-Navarro, I., van de Ven, G., et al., 2016, MNRAS, 463, 3220

Maraston, C., 2005, MNRAS, 362, 799

Marigo, P., Girardi, L., Bressan, A., Groenewegen, M. A. T., Silva, L., Granato, G. L., 2008, A\&A, 482, 883

Martín-Navarro I., La Barbera F., Vazdekis A., Falcón-Barroso J., Ferreras I., 2015a, MNRAS, 447, 1033 (MN15a)

Martín-Navarro I., et al., 2015b, ApJ, 806, L31 (MN15b) 
Martín-Navarro I., La Barbera F., Vazdekis A., Ferré-Mateu A., Trujillo I., Beasley M. A., 2015c, MNRAS, 451, 1081 (MN15c)

Martín-Navarro, I., Vazdekis, A., Falcón-Barroso, J., La Barbera, F., YÄśld Äśr Äśm, A., van de Ven, G., 2018, MNRAS, 475, 3700

Martín-Navarro I., et al., 2019, A\&A, 626, A124

McConnell N. J., Lu J. R., Mann A. W., 2016, ApJ, 821, 39

Milone, A. de C., Sansom, A. E., Sánchez-Blázquez, P., MNRAS, 2011, 414, 1227

Modigliani, A., Goldoni, P., Royer, F., et al. 2010, in Society of Photo-Optical Instrumentation Engineers (SPIE) Conference Series, Vol. 7737, Observatory Operations: Strategies, Processes, and Systems III, ed. D. R. Silva, A. B. Peck, \& T. Soifer

Navarro-González, J., Ricciardelli, E., Quilis, V., Vazdekis, A., 2013, MNRAS, 436, 3507

Newman, A. B., Smith, R. J., Conroy, C., Villaume, A., van Dokkum, P., 2017, ApJ, 845, 157

Oogi, T., Habe, A., Ishiyama, T., 2016, MNRAS, 456, 3000

Oser L., Ostriker J. P., Naab T., Johansson P. H., Burkert A., 2010, ApJ, 725, 2312

Oser, L., Naab, T., Ostriker, J. P., Johansson, P. H., 2012, ApJ, 744, 6

Padoan P., Nordlund A., 2002, ApJ, 576, 870

Parikh, T., et al., 2018, MNRAS, 477, 3954P

Pasquali, A., Smith, R., Gallazzi, A., De Lucia, G., Zibetti, S., Hirschmann, M., Yi, S. K., 2019, MNRAS, 484, 1702

Pietrinferni, A., Cassisi, S., Salaris, M., Castelli, F., 2006, ApJ, 642,797

Rayner, J. T., Toomey, D. W., Onaka, P. M., Denault, A. J., Stahlberger, W. E., Vacca, W. D., Cushing, M. C., Wang, S., 2003, PASP, 115, 362

Röck, B., Vazdekis, A., Ricciardelli, E., Peletier, R. F., Knapen, J. H., Falcón-Barroso, J., 2016, A\&A, 589, 73

Röck, B., Vazdekis, A., La Barbera, F., Peletier, R. F., Knapen, J. H., Allende-Prieto, C., Aguado, D. S., 2017, MNRAS, 472, 361

Rosani, G., Pasquali, A., La Barbera, F., Ferreras, I., Vazdekis, A., 2018, MNRAS, 476, 5233

Salpeter, E. E., 1955, ApJ, 121, 161

Sánchez-Blázquez, P., et al., 2006, MNRAS, 371, 703

Sánchez-Blázquez, P., Forbes, D. A., Strader, J., Brodie, J., Proctor, R., 2007, MNRAS, 377, 759

Saracco, P., Longhetti, M., Gargiulo, A., 2011, MNRAS, 412, 2707

Sarzi, M., Spiniello, C., La Barbera, F., Krajnović, D., van den Bosch, R., 2018, MNRAS, 478, 4084S

Schönebeck, F., Puzia, T. H., Pasquali, A., Grebel, E. K., KisslerPatig, M., Kuntschner, H., Lyubenova, M., Perina, S., 2014, A\&A, 572, 13

Serven, J., Worthey, G., Briley, M. M., 2005, ApJ, 627, 754

Smith, R. J., Lucey, J. R., 2013, MNRASL, 434, 1964

Smith, R. J., Alton, P., Lucey, J. R., Conroy, C., Carter, D., 2015, MNRAS, 454, 71 (SAL15)

Smith, R. J., Lucey, J. R., Conroy, C., 2015, MNRASL, 449, 3441

Spolaor, M., Proctor, R. N., Forbes, D., 2009, ApJ, 691, 138

Spiniello, C., Trager, S. C., Koopmans, L. V. E., Chen, Y. P., 2012, ApJ, 753, L32

Spiniello, C., Trager, S. C., Koopmans, L. V. E., Conroy, C., 2014, MNRAS, 438, 1438

Spiniello, C., Barnabé, M., Koopmans, L. V. E., Trager, S. C., 2015, ApJ, 452, L21 (SBK15)

Spinrad H., Taylor B. J., 1971, ApJS, 22, 445

Thomas D., Maraston C., Bender R., 2003, MNRAS, 339, 897

Tortora, C., Romanowsky, A. J., Napolitano, N. R., 2013ApJ, 765,8

Trager, S. C., Worthey, G., Faber, S. M., Burstein, D., González, J. J., 1998, ApJS, 116, 1

Treu T., Auger M. W., Koopmans L. V. E., Gavazzi R., Marshall
P. J., Bolton A. S., 2010, ApJ, 709, 1195

Trujillo, I., Conselice, C. J., Bundy, K., Cooper, M. C., Eisenhardt, P., Ellis, R. S., 2007, MNRAS, 382, 109

Trujillo, I., Ferré-Mateu, A., Balcells, M., Vazdekis, A., SánchezBlázquez, P.,2014, ApJ, 780, L20

Valdes, F., Gupta, R., Rose, J. A., Singh, H. P., Bell, D. J., 2004, ApJS, 152, 251

van Dokkum P. G., et al., 2008, ApJ, 677, L5

van Dokkum P. G., Conroy C., 2010, Nature, 468, 940

van Dokkum, P., Conroy, C., Villaume, A., Brodie, J., Romanowsky, A. J., 2017, ApJ, 841, 68V

Vijayaraghavan, R., Gallagher, J. S., Ricker, P. M., 2015, MNRAS, 447, 3623

Vaughan, S. P., Davies, R. L., Zieleniewski, S., Houghton, R. C. W., 2018, MNRAS, 479, 2443V

Vazdekis, A., Casuso, E., Peletier, R. F., Beckman, J. E., 1996, ApJS, 106, 307

Vazdekis A., Peletier R. F., Beckman J. E., Casuso E., 1997, ApJS, 111, 203

Vazdekis, A., Cenarro, A. J., Gorgas, J., Cardiel, N., Peletier, R. F., 2003, MNRAS, 340, 1317

Vazdekis, A., Ricciardelli, E., Cenarro, A. J., Rivero-González, J. G., Díaz-Garcá, L. A., Falcón-Barroso, J., 2012, MNRAS, 424,157

Vazdekis, A., Coelho, P., Cassisi, S., Ricciardelli, E., FalcónBarroso, J., Sánchez-Blázquez, P., La Barbera, F., Beasley, M. A., Pietrinferni, A., 2015, MNRAS, 449, 1177 (V15)

Vazdekis, A., Koleva, M., Ricciardelli, E., Röck, B., FalcónBarroso, J., 2016, MNRAS, 463, 3409

Vernet, J., et al., 2011, A\&A, 536, A105

Villaume, A., Brodie, J., Conroy, C., Romanowsky, A. J., van Dokkum, P., 2017, ApJ, 850, 14

Wang, L., Yang X., Shen, S., Mo H. J., van den Bosch F. C., Luo W., Wang, Y., Lau E.T., Wang Q. D., Kang X., Li R., 2014, MNRAS, 439, 611

Weidner, C., Ferreras, I., Vazdekis, A., La Barbera, F., 2013, MNRAS, 435, 2274

Yang X., Mo H. J., van den Bosch F. C., Jing Y.P., 2005, MNRAS, 356,1293

Yang X., Mo H. J., van den Bosch F. C., Pasquali A., Li C., Barden M., 2007, ApJ, 671, 153

Zieleniewski S., Houghton R. C. W., Thatte N., Davies R. L., Vaughan S. P., 2017, MNRAS, 465, 192

\section{APPENDIX A: THE ENVIRONMENT OF XSGS}

In order to assess the host environment of our targets, we matched the list of XSGs with the group catalogue of Wang et al. (2014), obtained from SDSS Data Release 7 (DR7), using the procedure of Yang et al. (2007). In short, the adaptive halo-based group finder of Yang et al. (2007) is applied to all galaxies in the Main Galaxy Sample of the New York University Value-Added Galaxy Catalogue (Blanton et al. 2005) for DR7 (Abazajian et al. 2009), in the redshift interval $0.01 \leqslant z \leqslant 0.20$, with a redshift completeness $\mathrm{C}_{z}>0.7$, and an extinction corrected apparent magnitude brighter than $r=17.72$ mag. Galaxy stellar masses are computed using the relations between stellar mass-to-light ratio and colour from Bell et al. (2003). The algorithm of Yang et al. (2007) is based on the traditional FOF method with small linking lengths in redshift space to arrange galaxies into groups and estimate the group characteristic stellar mass and luminosity. In the first iteration, the adaptive halo-based group finder uses a constant massto-light ratio of $500 h \mathrm{M}_{\odot} / \mathrm{L}_{\odot}$ to evaluate a tentative halo 
Table A1. Environmental properties of XSGs. Column 1 gives the galaxy number in our sample, column 2 reports if the galaxy is classified as a central (CEN) or satellite (SAT) in the group catalogue (see the text), while column 3 reports the halo mass of the parent halo where the galaxy resides.

\begin{tabular}{|c|c|c|}
\hline $\begin{array}{c}\text { XSG\# } \\
\text { (1) }\end{array}$ & $\begin{array}{c}\text { hierarchy } \\
\text { (2) }\end{array}$ & $\begin{array}{c}\log 10\left(\mathrm{M}_{\mathrm{h}}\right) \\
\left(M_{\odot}\right) \\
(3)\end{array}$ \\
\hline 1 & $\mathrm{CEN}$ & 14.00 \\
\hline 2 & SAT & 14.35 \\
\hline 6 & CEN & 13.85 \\
\hline 7 & SAT & 13.78 \\
\hline 8 & CEN & 14.33 \\
\hline 9 & CEN & 14.45 \\
\hline 10 & CEN & 13.52 \\
\hline
\end{tabular}

mass for each group. This mass is later used to estimate the size and velocity dispersion of the group halo, which in turn define group membership in redshift space. Next, a new iteration starts, in which the group characteristic luminosity and stellar mass are converted into halo mass through the halo occupation model of Yang et al. (2005). This procedure is reiterated until no more changes arise in group membership. Additional outputs of the algorithm are the group virial radius, the satellite projected distance from the luminosityweighted centre of the group, and the distinction of group members between centrals (the galaxies with largest stellar mass) and satellites.

From the above mentioned group catalogue, we retrieved information about hierarchy and host halo mass for our targets (see Tab. A1). Most of the XSGs, i.e. XSG1, XSG6, XSG8, XSG9, and XSG10 are classified as central galaxies, while XSG2 and XSG7 are satellites, with a projected distance of $0.45 R_{\text {vir }}$ and $0.08 R_{\text {vir }}$ from the luminosity-weighted centre of their parent group, respectively. For what concerns XSG7, in practice this system is hosted by a group with two bright galaxies at its center, one of them being XSG7 and the other being a galaxy with similar luminosity (classified as group central). Therefore, although classified as satellite, the nature of XSG7 is uncertain. Having stellar masses very similar to those of the brightest group galaxies of their parent groups (with stellar mass differences $\lesssim 0.15$ dex), both XSG2 ans XSG7 might have been centrals of recently infallen groups. To test this hypothesis, we also analyzed the phase-space of galaxies in the parent groups of XSG2 and XSG7 (using the same approach as in Pasquali et al. 2019), finding no clear evidence of multiple components. Notice that, as shown by the numerical simulations of Vijayaraghavan et al. (2015), accreted groups may be not detectable in phase space a few Gyr after infall, implying that both XSG2 and XSG7 could still have been centrals for most of their evolutionary history. In such case, all radial profiles (and in particular IMF gradients) of the XSGs would be representative of the population of central ETGs at high-mass end. For what concerns parent halo mass, XSG1, XSG2, XSG8, and XSG9, reside in hosts with a halo mass between $10^{14}$ and $10^{14.5} \mathrm{M}_{\odot} h^{-1}$, whereas XSG6, XSG7 and XSG10 belong to environments in the range $10^{13.5}-10^{14} \mathrm{M}_{\odot} h^{-1}$.

\section{APPENDIX B: COMPARISON OF SDSS AND X-SHOOTER CENTRAL SPECTRA}

All galaxies in our sample have SDSS spectroscopy available, in the optical spectral range, covering a fiber circular aperture of radius 1.5". Fig. B1 shows a representative comparison of X-Shooter and SDSS spectra for three galaxies in our sample (XSG6, XSG8, and XSG10). In order to mimic the SDSS circular aperture, the X-Shooter longslit spectra have been extracted by weighting each point along the slit with a factor $\pi R$, where $R$ is the distance from the photometric center of the galaxy. Both X-Shooter and SDSS spectra have been corrected for Galactic reddening, with wavelengths given at restframe, in the air system. Fig. B1 shows an excellent agreement (at few percent level) between the SDSS and X-Shooter spectra, despite the fact that we are comparing fiber to long-slit data. As expected (because of the higher spectral resolution), the X-Shooter spectra have far better quality, than the SDSS ones, in the regions strongly affected by telluric/airglow sky lines (e.g. in the CaT region, at $\lambda \sim 8600 \AA$ ). The insets in the Figure zoom out the wavelength window, where the UVB and VIS $\mathrm{X}$-Shooter spectra have been merged during the reduction process, at $\lambda \sim 5400 \AA$ restframe (see LB16). This region is potentially affected by the temporal variation of the instrumental throughput due to the UVB/VIS dichroic element (see, e.g., Schönebeck et al. 2014). The comparison of SDSS and X-Shooter spectra shows a very good agreement also in the dichroic region. In particular, we verified that the linestrengths of Fe5270 and Fe5335 spectral features (which enter our analysis, and fall in the dichroic region) are fully consistent (at 1 sigma level) between the two data-sets, further confirming the reliability of our data reduction procedure.

\section{APPENDIX C: SURFACE PHOTOMETRY}

In order to characterize the light distribution of the XSGs, and in particular the scale radius of their light profiles, we have used the software 2DPHOT (La Barbera et al. 2008) to fit the SDSS r-band images of all galaxies with twodimensional, PSF-convolved, models. We have considered (i) single component Sérsic models, and (ii) models with a Sérsic bulge, plus an exponential (disc) component (hereafter $\mathrm{B}+\mathrm{D})$. Galaxy images, as well as residual maps after subtracting the best-fitting models, are shown in Fig. C1. Relevant parameters of the fits, namely the effective radius of the single Sérsic and $B+D$ fits, $R_{e, S}$ and $R_{e, T}{ }^{18}$, respectively, the axis ratio of the single-component fits, $\mathrm{b} / \mathrm{a}$, the total magnitudes of the Sérsic and $\mathrm{B}+\mathrm{D}$ models, $\mathrm{m}_{\mathrm{T}, \mathrm{S}}$ and $\mathrm{m}_{\mathrm{T}, \mathrm{B}+\mathrm{D}}$, respectively, and the total light fraction in the bulge, $\mathrm{B} /(\mathrm{B}+\mathrm{D})$, are reported in Tab. $\mathrm{C} 1$.

Fig. C1 shows that while, in general, a single Sérsic component describes reasonably well the XSGs' light distribution, the Sérsic fits are not perfect, leaving either small central residuals (see, e.g., the case of XSG10) or largescale faint features in the model-subtracted images (see, e.g.,

18 The $\mathrm{R}_{\mathrm{e}, \mathrm{T}}$ is computed by constructing a growth light curve from the best-fitting $\mathrm{B}+\mathrm{D}$ model, and deriving the radius enclosing half of the total light of the model. 


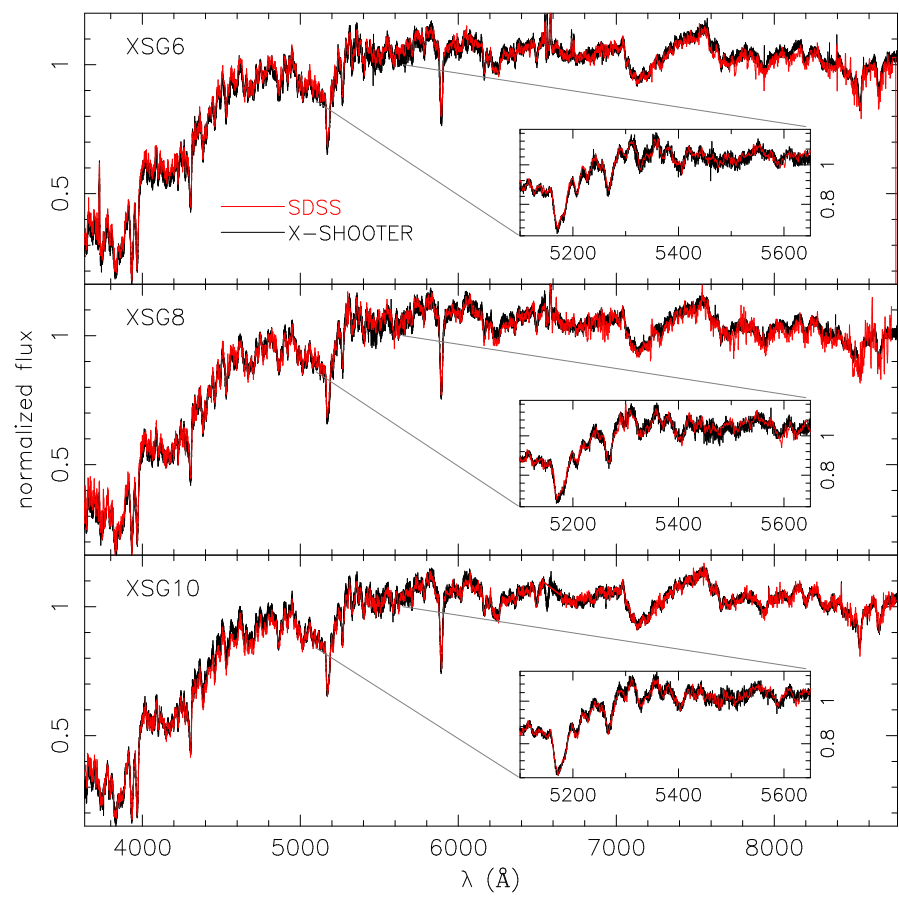

Figure B1. Examples of X-Shooter (black) and SDSS (red) optical spectra, for three galaxies in our sample. The X-Shooter spectra have been extracted to mimic the same circular aperture as the SDSS fiber (see the text). A very good agreement is found, even in the region from 5200 to $5600 \AA$ restframe (see the inset panels), which is corrected for the effect of the X-Shooter dichroic (see the text)

XSG8). Most of the residual features disappear when adopting a second component, through a $\mathrm{B}+\mathrm{D}$ decomposition, with the exception of XSG6, for which both Sérsic and B+D fits give similar residual maps. Notice also that the second component is significant, accounting for a fraction $\gtrsim 50 \%$ of the total galaxy light in most systems (XSG1, XSG2, XSG7, XSG8, and XSG9). We point out here that the lack of significant rotation in all the XSG's indicates that the second component describes actually an halo envelope, rather than a true (kinematical) disc. This result is consistent with the fact that most of XSGs are central group galaxies (see App. A), that may have built up their extended envelopes through several minor mergers/smooth accretion. We also find that most of the XSGs are round objects, with an axis ratio 0.8-0.9 (i.e. an E1-E2 morphological type), while XSG2 and XSG8 have a flatter shape $(\mathrm{b} / \mathrm{a} \sim 0.6)$, corresponding to an E4 type.

Tab. C1 shows that for more than half of the XSGs the effective radius depends significantly on the fitting method. In fact, while for XSG1, XSG2, and XSG10, the difference between $R_{e, S}$ and $R_{e, T}$ is within $\sim 20 \%$, for XSG6, XSG7, XSG8, and XSG9, the $\mathrm{R}_{\mathrm{e}, \mathrm{S}}$ is significantly larger, by a factor about two, than $R_{e, T}{ }^{19}$. The effective radius of the bulge

19 Notice that XSG1 and XSG10 are the only two galaxies with $R_{e, S}<R_{e, T}$. For XSG10, this is due to the fact that the B+D model consists of an extended bulge component (with $R_{e, B} \sim$ $\left.6.5 \mathrm{kpc}>R_{\mathrm{e}, \mathrm{S}}\right)$ and a very small, faint, disk $\left(\mathrm{R}_{\mathrm{e}, \mathrm{D}} \sim 0.5 \mathrm{kpc}\right)$. Hence, the total $R_{e, T}$ is driven by $R_{e, B}$. For XSG1, the disk com- component does also show a wide range of values, from $\sim 2$ to $\sim 8 \mathrm{kpc}$, with a characteristic (mean) value of $\sim 3.9 \mathrm{kpc}$. Notice that this characteristic scale matches the radial range covered by our radially binned X-Shooter spectra.

\section{APPENDIX D: IMF PROFILES VERSUS $R / \mathrm{R}_{\mathrm{E}}$.}

As reported in Sec. 5.1, while IMF profiles of the XSGs appear to be all similar when plotted versus galactocentric distance in physical units, the profiles do differ significantly when normalizing distances by the effective radius, $R_{e}$. This is shown in Fig. D1, where we plot the best-fitting IMF slope, for our reference fitting method, versus $R / R_{e}$, considering effective radii from single Sérsic fits (top panel), for the bulge component only (middle panel), and from $\mathrm{B}+\mathrm{D}$ models. As discussed in Sec. 3, most X-Shooter galaxies exhibit an extended outer envelope making the estimate of $R_{e}$ strongly dependent on the adopted method. In particular, XSG6, XSG8, and XSG9 have single Sérsic and B+D effective radii significantly larger than those of the other galaxies. Hence, their IMF profiles, when plotted as a function of $\mathrm{R} / \mathrm{R}_{\mathrm{e}}$, appear more concentrated than those of XSG1, XSG2, XSG7, and XSG10 (see top and bottom panels in Fig. D1). This implies, in turn, that within a given fraction of $R_{e}$, the (luminosity-weighted) IMF slope and mass-tolight ratio of the XSG's would show a large scatter.

\section{APPENDIX E: BEST-FITTING AND OBSERVED LINE-STRENGTHS.}

Fig. E1 compares observed and best-fitting IMF-sensitive spectral indices of all X-Shooter spectra, for our reference fitting method. In Fig. E2, we also show fitting results for the total-metallicity indicator $[\mathrm{MgFe}]^{\prime}$, as well as for $\mathrm{H} \beta_{\mathrm{o}}$ and $\mathrm{H} \gamma_{\mathrm{F}}$ Balmer lines. Notice that all best-fitting indices are corrected to a broadening of $300 \mathrm{~km} \mathrm{~s}^{-1}$ velocity dispersion, following a similar procedure as in LB16 and LB17 (i.e. using best-fitting stellar population models to evaluate the a broadening correction to line-strengths). Results are shown for our reference fitting method (method A), but for $\mathrm{H} \beta_{\mathrm{O}}$ and $\mathrm{H} \gamma_{\mathrm{F}}$, as Balmer lines are only included in the 2SSP fitting procedure. Hence, for $\mathrm{H} \beta_{\mathrm{o}}$ and $\mathrm{H} \gamma_{\mathrm{F}}$, Fig. E2 overplots best-fitting indices from method D (see Tab. 2).

Fig. E1 shows that we fit reasonably well all spectral indices, although some discrepancies between models and observations are actually found:

- TiO1 shows a steeper trend in the data, with respect to models. This might be due to (i) the effect of non-solar abundance ratios, which is not captured by our modeling approach; (ii) the fact that $\mathrm{TiO} 1$ is a very broad feature, whose radial gradient might be affected by sky-continuum subtraction; (iii) the extrapolation of the feature in the high metallicity regime (see also LB16);

- aTiO shows some offset/tilt with respect to observations (in particular for XSG1, XSG7, and XSG9). Notice that aTiO is a very broad feature, even broader than the

ponent is significant, and more extended $\left(\mathrm{R}_{\mathrm{e}, \mathrm{D}} \sim 10 \mathrm{kpc}\right)$ than the bulge $\left(R_{e, B} \sim 1.8 \mathrm{kpc}\right)$, making $R_{e, T}$ larger than $R_{e, S}$. 

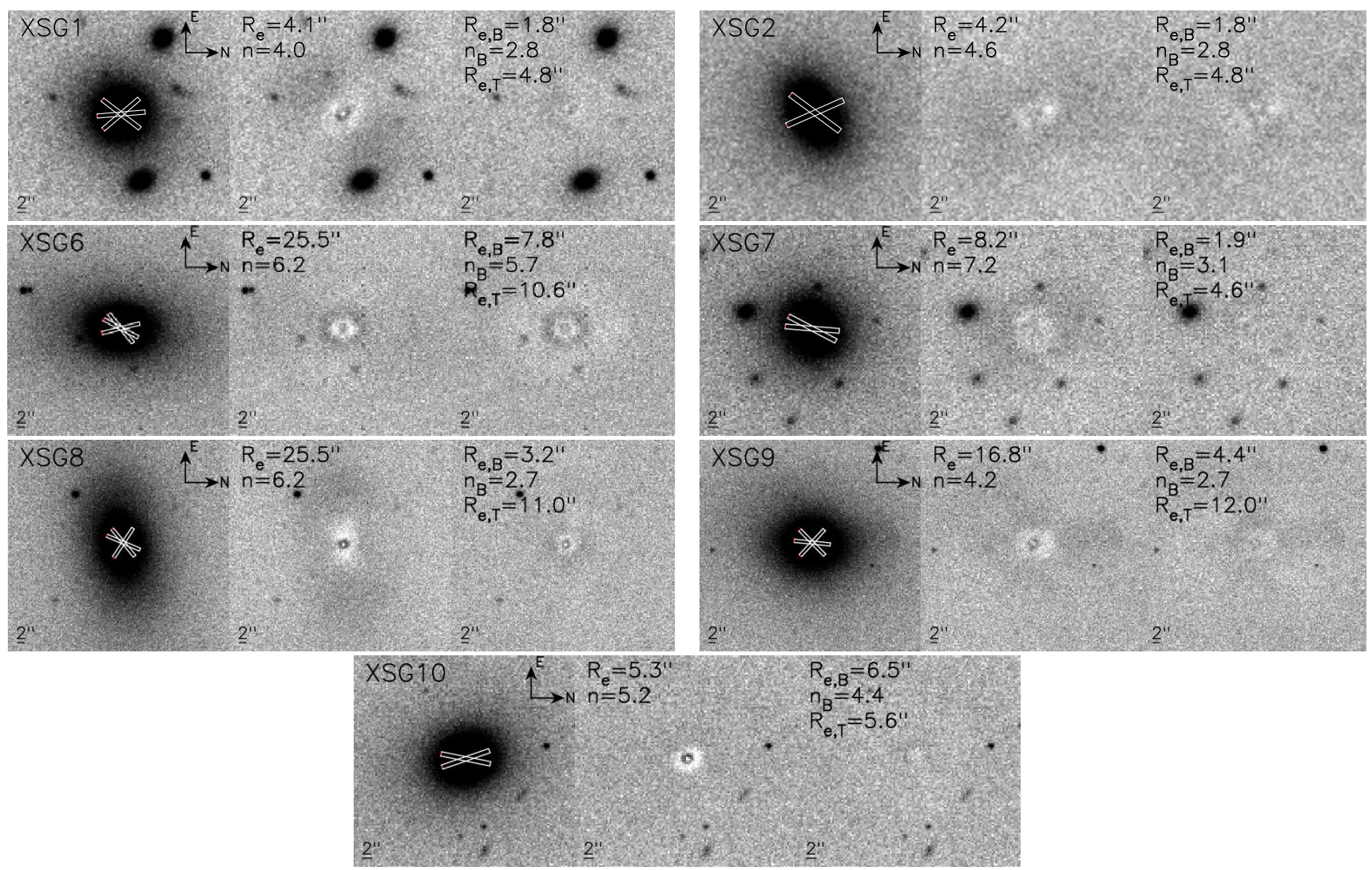

Figure C1. Two-dimensional surface brightness decomposition of the SDSS r-band images of our massive galaxies targeted with XShooter (see Tab. 1). From left to right, and top to bottom, galaxies are shown in the same order as listed in Tab. 1. For each galaxy, the Figure plots three horizontal panels, showing the r-band SDSS galaxy image (left), with the galaxy ID on the top-left corner; the residual map, normalized to the expected noise in each pixel, after subtracting the best-fitting Sersic model (middle); the same as in the middle panel, but for the (Sersic-)bulge plus disc (B+D) decomposition (right). In the top-left corner of each middle sub-panel, we report the effective radius, $\mathrm{R}_{\mathrm{e}, \mathrm{S}}$, and the shape parameter, $\mathrm{n}_{\mathrm{S}}$, of the best-fitting Sersic models. In the right sub-panels, we report the effective radius, $R_{e, B}$, and the shape parameter, $n_{B}$, of the best-fitting bulge component, as well as the half-light radius of the $B+D$ model, $\mathrm{R}_{\mathrm{e}, \mathrm{T}}$. The relevant best-fitting parameters are summarized in Tab. C1.

Table C1. Structural parameters of XSG galaxies in $\mathrm{r}$ band. Column 1 is the XSG label. Columns 2, 3, 4, and 5, report the best-fitting Sersic parameters, namely the effective radius, $\mathrm{R}_{\mathrm{e}, \mathrm{S}}$, the axis ratio $\mathrm{b} / \mathrm{a}$, the shape parameter $\mathrm{n}_{\mathrm{S}}$, and total magnitude $\mathrm{m}_{\mathrm{T}, \mathrm{S}}$. Columns 6 , $7,8,9,10$ provide the most relevant best-fitting parameters of the $\mathrm{B}+\mathrm{D}$ decomposition, i.e. the effective radius and Sersic index of the bulge component, $R_{e, S}$ and $n_{B}$, the total (i.e. $\left.B+D\right)$ effective radius, $R_{e, T}$, the bulge luminosity fraction, $B /(B+D)$, as well as the total magnitude, $\mathrm{m}_{\mathrm{T}, \mathrm{B}+\mathrm{D}}$, respectively.

\begin{tabular}{|c|c|c|c|c|c|c|c|c|c|}
\hline $\begin{array}{c}\text { XSG\# } \\
\text { (1) }\end{array}$ & $\begin{array}{c}\mathrm{R}_{\mathrm{e}, \mathrm{S}} \\
(\mathrm{kpc}) \\
(2)\end{array}$ & $\begin{array}{l}\mathrm{b} / \mathrm{a} \\
(3)\end{array}$ & $\begin{array}{l}\mathrm{n}_{\mathrm{S}} \\
(4)\end{array}$ & $\begin{array}{c}\mathrm{m}_{\mathrm{T}, \mathrm{S}} \\
(\mathrm{mag}) \\
(5)\end{array}$ & $\begin{array}{c}\mathrm{R}_{\mathrm{e}, \mathrm{B}} \\
(\mathrm{kpc}) \\
(6)\end{array}$ & $\begin{array}{l}\mathrm{n}_{\mathrm{B}} \\
(7)\end{array}$ & $\begin{array}{c}\mathrm{R}_{\mathrm{e}, \mathrm{T}} \\
(\mathrm{kpc}) \\
(8)\end{array}$ & $\begin{array}{c}\mathrm{B} /(\mathrm{B}+\mathrm{D}) \\
(9)\end{array}$ & $\begin{array}{c}\mathrm{m}_{\mathrm{T}, \mathrm{B}+\mathrm{D}} \\
(\mathrm{mag}) \\
(10)\end{array}$ \\
\hline 1 & 4.1 & 0.90 & 4.02 & 14.40 & 1.8 & 2.78 & 4.84 & 0.51 & 14.43 \\
\hline 2 & 4.2 & 0.63 & 4.58 & 14.61 & 1.6 & 3.51 & 3.95 & 0.54 & 15.09 \\
\hline 6 & 20.4 & 0.75 & 6.81 & 13.32 & 7.8 & 5.73 & 10.6 & 0.70 & 13.83 \\
\hline 7 & 8.2 & 0.84 & 7.15 & 14.25 & 1.9 & 3.14 & 4.6 & 0.55 & 14.45 \\
\hline 8 & 25.5 & 0.63 & 6.15 & 12.98 & 3.2 & 2.66 & 11.0 & 0.39 & 13.25 \\
\hline 9 & 16.8 & 0.84 & 4.16 & 13.22 & 4.4 & 2.66 & 12.0 & 0.40 & 13.52 \\
\hline 10 & 5.3 & 0.85 & 5.20 & 14.17 & 6.5 & 4.43 & 5.63 & 0.93 & 14.25 \\
\hline
\end{tabular}



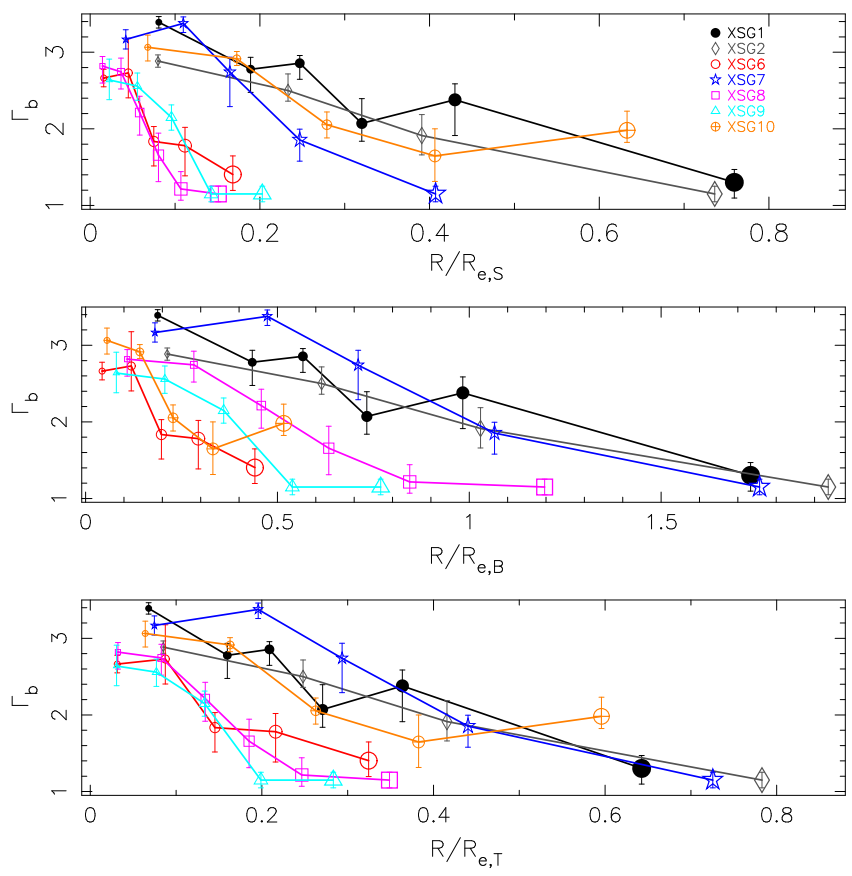

Figure D1. Same as Fig. 2 but rescaling the galactocentric distance, $\mathrm{R}$, by the galaxy effective radius. We consider (top) effective radii from single Sérsic fits of galaxy images, (middle) effective radii of the bulge component only (from $\mathrm{B}+\mathrm{D}$ decomposition), and (bottom) "total" effective radii from $\mathrm{B}+\mathrm{D}$ models (see Sec. 3 for details). Effective radii for different galaxies are reported in Tab. $\mathrm{C} 1$. Notice the different $\mathrm{x}$-axis scale of the three panels, due to differences in $R_{e}$ among different methods.

TiO's. Hence sky-continuum subtraction/flux calibration uncertainties (at one percent level) can significantly affect its radial behaviour. Moreover, as discussed in LB16, the competing effect of IMF and $[\mathrm{Z} / \mathrm{H}]$ does not make this feature an IMF-indicator as good as other features.

- Remarkably, we are able to fit all four Na lines, simultaneously, at different radial positions. This confirms the finding of LB17, that the combined effect of a bottom-heavy IMF and overabundant $[\mathrm{Na} / \mathrm{Fe}]$ is crucial to describe Na features. Nevertheless, we notice that for most galaxies, NaI2.21 is underpredicted by our models. This is somewhat expected, as theoretical stellar spectra used to construct Na-EMILES models do not cover temperatures cooler than $\mathrm{T}_{\text {eff }}=3500 \mathrm{~K}$ (see LB17). Hence, for stars with $\mathrm{T}_{\text {eff }}<3500 \mathrm{~K}$ in the empirical stellar libraries (see Sec. 3), we apply differential corrections for $[\mathrm{Na} / \mathrm{Fe}]$ by assuming $\mathrm{T}_{\text {eff }}=3500 \mathrm{~K}$. At the SSP level, this implies that NIR Na features (and in particular NaI2.21) might be underestimated by $\sim 0.1-0.2$ dex (see LB17), which is fully consistent with what seen in the bottom panels of Fig. E1. Moreover, since $[\mathrm{C} / \mathrm{Fe}]$ is over-abundant in massive ETGs, the underestimation of NaI2.21 might also due to a significant contribution of carbon to NaI2.21, as discussed in Röck et al. (2017).

We also notice that Balmer lines are well matched by our 2SSP fitting scheme, implying that our IMF results are also insensitive to age constraints. Indeed, the only galaxies with some evidence of age gradients are XSG6 and XSG10, where $H \beta_{\text {o }}$ increases significantly with galacto-centric dis- tance. The presence of a (negative) age gradient for XSG6 is also confirmed by our reference fitting method A (see the age of XSG6 decreasing outwards in panel e of Fig. 4), where none of the Balmer lines is included in the fitting procedure, as well as by the presence of significant emission lines in this galaxy at all radii (see Appendix F). For XSG10, the situation is less clear, as this galaxy does not show any significant emission (see Appendix F), and method A does not provide any significant age gradient (see panel e of Fig. 4). Therefore, for XSG10, the $\mathrm{H} \beta$ radial gradient might be reflecting an IMF, rather than an age, variation (in fact, $\mathrm{H} \beta$ o becomes weaker with increasing IMF slope; see LB13). Whatever is causing the radial trend of $\mathrm{H} \beta_{\mathrm{o}}$ for XSG10, the key point for the present work is that for this galaxy, methods A and $\mathrm{D}$ provide very consistent IMF radial variations. More in general, the fact that different combinations of spectral indices (Tab. 2) give similar IMF radial trends for all galaxies, proves the robustness of our results, despite some unavoidable uncertainty in (state-of-art) stellar population models.

\section{APPENDIX F: EMISSION LINES IN THE X-SHOOTER SPECTRA.}

Some X-Shooter spectra of galaxies in our sample show emission contamination in the Balmer lines, and/or other weak emission lines in the optical spectral range. In order to characterize the nature of the emission, we have used the BPT (Baldwin, Philipps, Terlevich 1981) and WHAN (see Cid Fernandes et al. 2004 and references therein) diagnostic diagrams, fitting the relevant emission lines, i.e. $\mathrm{H} \beta,[\mathrm{OIII}] \lambda 5007,[\mathrm{NII}] \lambda 6548, \mathrm{H} \alpha$, and $[\mathrm{NII}] \lambda 6584$; the latter, though not explicitly used in the BPT/WHAN diagrams, is analyzed as it can affect the $\mathrm{H} \alpha$ line due to the small relative separation of $[\mathrm{NII}] \lambda 6584$ and $\mathrm{H} \alpha$. The fits are performed with a dedicated Python code, after subtracting the best-fitting model of the stellar component to each spectrum, within spectral windows large enough to ensure that both the emission line profiles and a significant portion of the continuum are included in the fitting range. The stellar component is modeled with a linear superposition of MILES SSP models, with varying age and metallicity, combined with CvD12a response functions to account for the effect of non-solar abundance ratios. The fits include a constant tilt in the residual continuum around the emission lines, and are performed by treating either (a) velocity, $\mathrm{v}_{\mathrm{em}}$, and velocity dispersion, $\sigma_{\mathrm{em}}$, as independent fitting parameters for each emission line; or (b) fixing $\mathrm{v}_{\mathrm{em}}$ and $\sigma_{\mathrm{em}}$ to be the same for all lines. We have verified that all the results reported here are independent of the assumptions on $\mathrm{vem}_{\mathrm{em}}$ and $\sigma_{\mathrm{em}}$.

Most galaxies in our sample (XSG2, XSG7, XSG9 and XSG10) are found to show negligible emission, as illustrated in Fig. F1, where we plot the residual flux (after subtracting the stellar component) in the spectral regions around the $\mathrm{H} \alpha$ line, for all radial bins. On the other hand, XSG1, XSG6, and XSG8, present detectable emission lines, whose ionization pattern can be characterized through the WHAN diagram, as shown in Fig. F2:

- XSG1 shows emission lines up to the largest radial bin probed, also detectable in the [OII] $\lambda 3726-3728$ and [SII] $\lambda 6716-6730$ lines. The WHAN diagram shows that the 


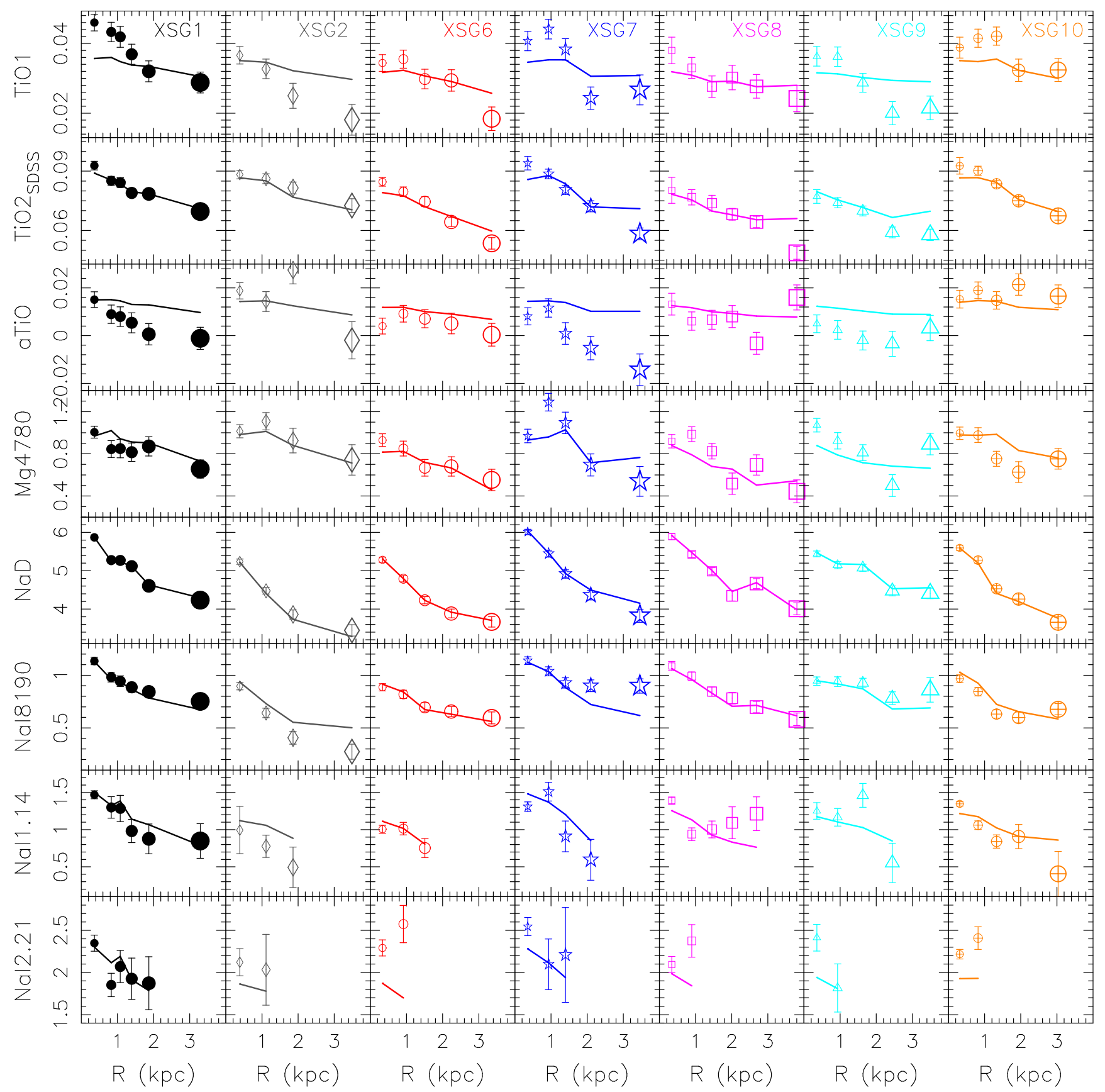

Figure E1. Observed and best-fitting line-strengths as a function of galactocentric distance, for our reference fitting scheme (method A; see Tab. 2). Observed line-strengths are plotted as symbols with error bars, while best-fitting indices are given by the solid lines. Only IMF-sensitive features are shown in the plot. Different galaxies are plotted from left to right (see labels in the top panels), while different indices are plotted along each column in the Figure, from top (TiO1) to bottom (NaI2.21). Line-strengths of different galaxies are plotted with different symbols (whose size increases with galactocentric distance) and colours, as in Fig. 2. Error bars denote $1 \sigma$ uncertainties.

galaxy is retired (emission line produced by hot postasymptotic giant branch stars) in the center and passive ${ }^{20}$ in the outer regions (see filled circles in Fig. F2);

20 Notice that, as discussed in Cid Fernandes et al. (2004), the separation between retired and passive galaxies is somewhat arbitrary, as both classes include similar objects. "Passive" galaxies are those objects for which emission lines are extremely weak, making their measurement less safe.
- XSG6 also has intense [OII] $\lambda 3726-3728$, [SII] $\lambda 6716-6730$ and $[\mathrm{OI}] \lambda 6300$ emission. The WHAN diagram points to a retired ionization pattern for all apertures (see open circles in the Figure);

- As XSG1, XSG8 also presents detectable [OII] $\lambda 3726-$ 3728 and [SII] $\lambda 6716-6730$ emission, at least in the most central radial bins. The WHAN diagram indicates shows a retired ionization pattern, but for the last measured aperture where the system is passive (see open squares in the Figure). 


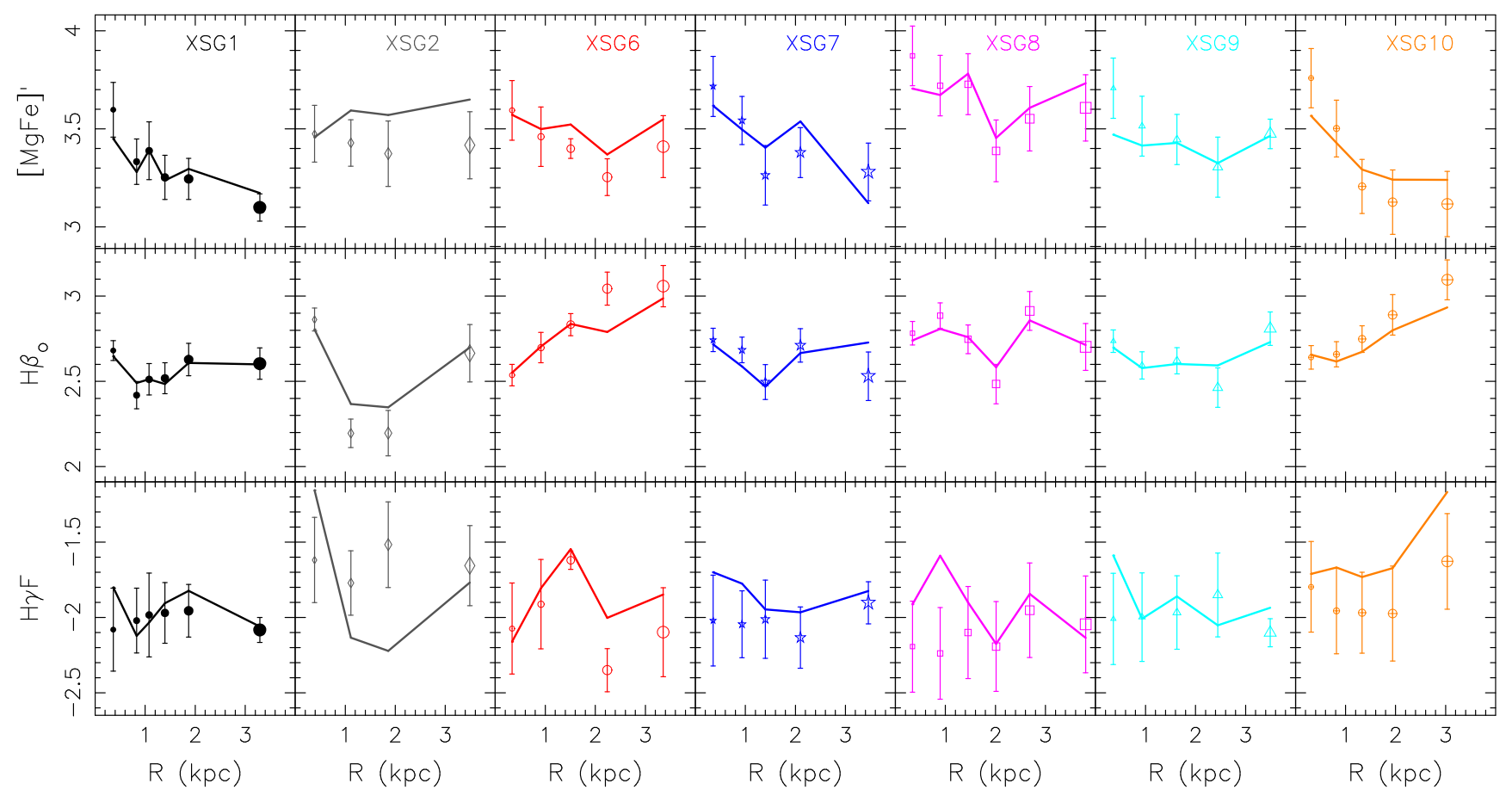

Figure E2. Same as Fig. E1 but for the total metallicity indicator $[\mathrm{MgFe}]^{\prime}$ (top row), and $\mathrm{H} \beta_{\mathrm{O}}$ and $\mathrm{H} \gamma_{\mathrm{F}}$ Balmer lines (middle and bottom rows, respectively). Notice that for $[\mathrm{MgFe}]^{\prime}$ we plot best-fitting line-strengths from our reference fitting scheme (as in Fig. E1), while for the Balmer lines we consider results for method D (see Tab. 2), as Balmer lines are not included in the fitting for the other methods.

Although we have only a few galaxies with emission, we notice that XSG1 has a more concentrated gas distribution compared to XSG6 and XSG8 where from inner to outer regions emission is always in the "retired" region of the WHAN diagram. Another way of interpreting is that since XSG6 and XSG8 also have much larger $R_{e}$ than XSG1, this trend may just be reflecting how gas traces stars.

We verified that all results reported above from the WHAN diagram are consistent with those from the BPT diagram, that is not shown here for brevity reasons. Hence, to summarize, XSG1, XSG6, and XSG8 show a ionization pattern that varies from retired to passive with increasing galactocentric distance, while the other XSGs do not show any measurable emission. Notice that, as discussed in the text, no significant dependence of IMF radial variations are found between galaxies with different ionization patterns.

This paper has been typeset from a TEX/LATEX file prepared by the author.

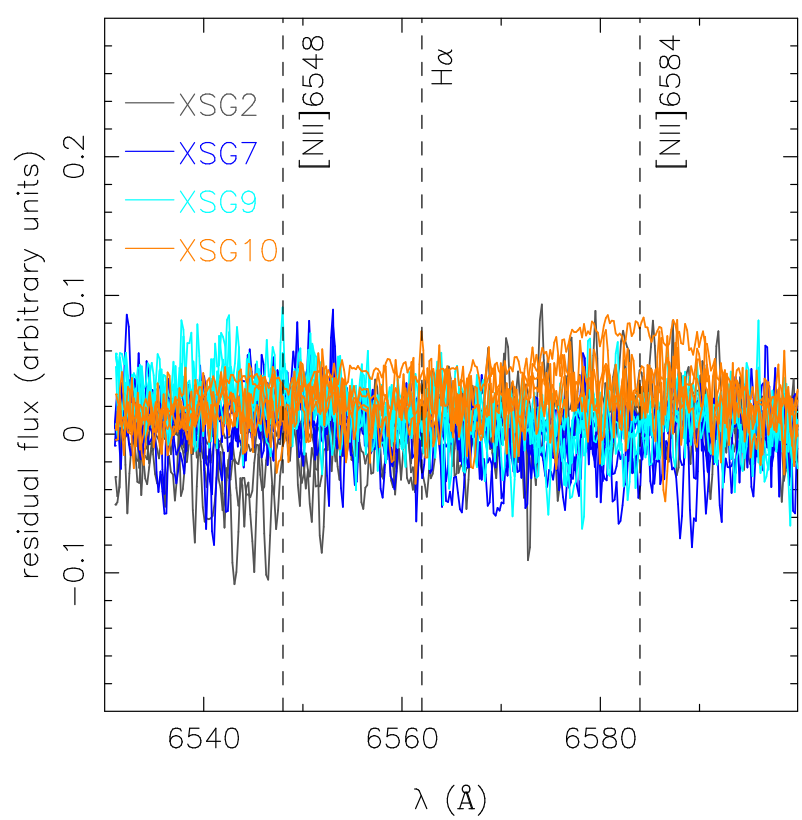

Figure F1. Residual spectra (after subtracting the best-fit stellar spectrum) for all radial bins of XSG2, XSG7, XSG9, and XSG10, in the restframe spectral window around the $\mathrm{H} \alpha$ line, from 6530 to $6600 \AA$. The position of the emission lines [NII] $\lambda 6548, \mathrm{H} \alpha$, and $[\mathrm{NII}] \lambda 6584$ are marked with dashed vertical lines. No emission pattern is detected for these galaxies. Different galaxies are plotted with the same colour coding as in Fig. 2. 


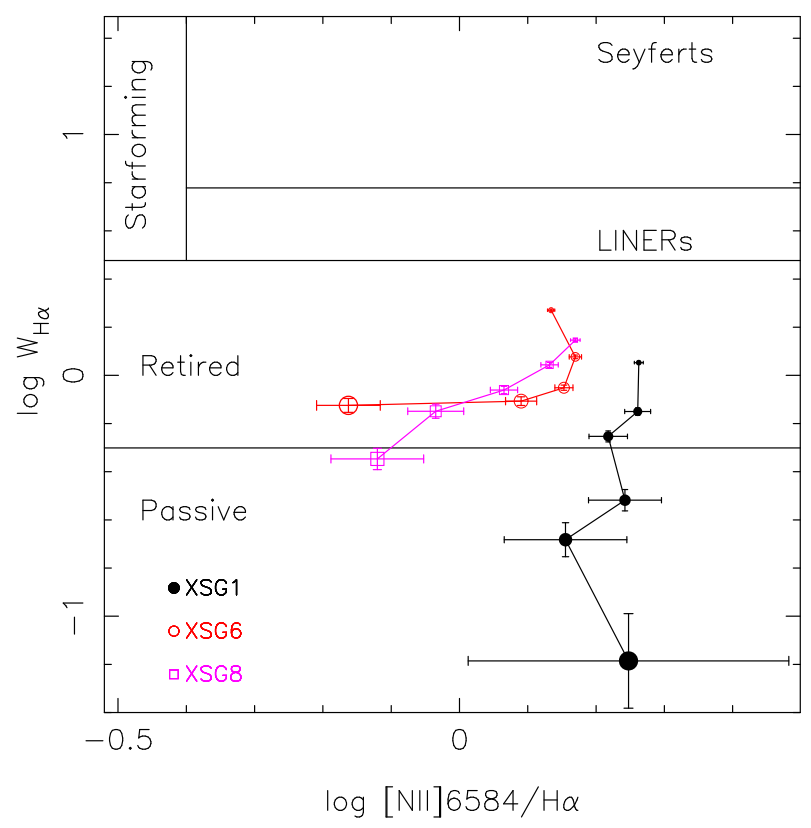

Figure F2. WHAN diagram for XSG1, XSG6, and XSG8. Each galaxy is plotted with the same symbol types and colours as in Fig. 2. Symbol sizes increase with galactocentric distance. For these galaxies, the ionization pattern tends to vary from retired in the center, to passive in the outskirts. For the other XSGs (XSG2, XSG7, XSG9, and XSG10), no emission is detectable in our spectra. 